\title{
Iron homeostasis related genes in rice
}

\author{
Jeferson Gross ${ }^{1,3}$, Ricardo José Stein ${ }^{2}$, Arthur Germano Fett-Neto ${ }^{1,2}$ and Janette Palma Fett ${ }^{1,2}$ \\ ${ }^{1}$ Universidade Federal do Rio Grande do Sul, Centro de Biotecnologia, Porto Alegre, RS, Brazil. \\ ${ }^{2}$ Universidade Federal do Rio Grande do Sul, Departamento de Botânica, Porto Alegre, RS, Brazil. \\ ${ }^{3}$ Botanical Institute of Ludwig-Maximilians-Universität, München, Germany.
}

\begin{abstract}
Iron is essential for plants. However, excess iron is toxic, leading to oxidative stress and decreased productivity. Therefore, plants must use finely tuned mechanisms to keep iron homeostasis in each of their organs, tissues, cells and organelles. A few of the genes involved in iron homeostasis in plants have been identified recently, and we used some of their protein sequences as queries to look for corresponding genes in the rice (Oryza sativa) genome. We have assigned possible functions to thirty-nine new rice genes. Together with four previously reported sequences, we analyzed a total of forty-three genes belonging to five known protein families: eighteen YS (Yellow Stripe), two FRO ( $\mathrm{Fe}^{3+}$-chelate reductase oxidase), thirteen ZIP (Zinc regulated transporter / Iron regulated transporter Protein), eight NRAMP (Natural Resistance - Associated Macrophage Protein), and two Ferritin proteins. The possible cellular localization and number of potential transmembrane domains were evaluated, and phylogenetic analysis performed for each gene family. Annotation of genomic sequences was performed. The presence and number of homologues in each gene family in rice and Arabidopsis is discussed in light of the established iron acquisition strategies used by each one of these two plants.
\end{abstract}

Key words: iron, rice, genome analysis, iron transporters, ferritin.

Received: February 15, 2003; Accepted: August 15, 2003.

\section{Introduction}

Iron is a key micronutrient for plants, taking part in redox centers of proteins essential for photosynthesis and respiration. Iron deficiency causes a metabolic imbalance deleterious to plant development (Briat, 1995). This problem is especially visible in calcareous soils, where the high $\mathrm{pH}$ dislocates the chemical balance towards the formation of iron insoluble complexes, leaving the cation sparsely available for plant uptake (Guerinot and Yi, 1994; Briat et al., 1995). However, in anaerobic conditions and acid $\mathrm{pH}$, as found in waterlogged soils, the ferrous form is stabilized and readily taken up by plants. When in excess, iron induces the production of hydroxyl radicals, the most potent oxidizing agents known. These radicals cause multiple damage to cellular structures, eventually leading to death (Guerinot and Yi, 1994; Briat et al., 1995). Therefore plants must balance iron concentration in a homeostatic way, providing the necessary amounts of the micronutrient and preventing the condition of internal cation excess (Briat and Lobréaux, 1997, Grusak et al., 1999).

Send correspondence to Janette Palma Fett. Universidade Federal do Rio Grande do Sul, Centro de Biotecnologia, Caixa Postal 15005, 91501-970 Porto Alegre, RS, Brazil. E-mail: jpfett@dna .cbiot.ufrgs.br.
The biotechnological manipulation of plant crops in a way to increase their capacity to adequate the iron content may result in yield improvement (Takahashi et al., 2001; Guerinot, 2001). Engineering plant crops for efficient iron nutrition is also justified because about $60 \%$ to $80 \%$ of the world population suffers in some manner from organic deficiencies of this micronutrient (World Health Organization, 2002). The increase in iron content in plant staple foods can serve as a vector for iron ingestion in target populations, minimizing nutritional problems (Vansuyt et al., 2000; Murrey-Kolb et al., 2002). In this regard, a better understanding of iron homeostasis, involving knowledge of the basic physiological processes of iron absorption, distribution and storage in plants, can serve as starting point for the biotechnological manipulation of crops (Grusak et al., 2002; Grotz and Guerinot, 2002). Plant iron homeostasis has been often analyzed by physiological and molecular genetics approaches. More recently, genomics also appears as an alternative for the understanding of this aspect of plant nutrition.

Traditional physiological studies gave special emphasis to the mechanisms for iron uptake from the soil. These have been classified in two distinct strategies (Marschner and Römheld, 1994). Strategy I is shared be- 
tween dicotyledoneous and non graminaceous monocotyledoneous species. Since iron in its ferric form is more soluble at low $\mathrm{pH}$, these plants acidify the rhizosphere by means of a $\mathrm{H}^{+}$-ATPase pump and release reductants/ chelators such as organic acids and phenolics (Römheld and Marschner, 1983; Römheld et al., 1984). The ferric ion is then chelated by the released compounds, as well as by soil bacteriosiderophores (Römheld and Marschner, 1983; Bar-Ness et al.,1992). Subsequently, chelated $\mathrm{Fe}^{3+}$ is reduced by a low iron-inducible enzymatic activity present in the plasma membrane of root epidermal cells (Moog and Brüggermann, 1994) and $\mathrm{Fe}^{+2}$ is then absorbed by a specific transporter (Fox et al., 1996). A different strategy to overcome the problem of low iron availability in the soil evolved in grasses (such as rice). The so called strategy II consists of releasing phytosiderophores, non-proteinogenic amino acids (structural derivatives of mugineic acid) that present mineral chelating properties (Sugiura and Nomoto, 1984; Römheld and Marschner, 1986). Depending on the mineral needs of the plant, these compounds are released to the rhizosphere, where they chelate the sparsely soluble $\mathrm{Fe}^{3+}$, making it more available for uptake (von Wirén et al., 1995). The $\mathrm{Fe}^{+3}$-phytosiderophore complex is readily absorbed by the plant, without need of extracellular reduction (Römheld and Marschner, 1986; von Wirén et al., 1995).

With this physiological model in mind, a new approach based on molecular genetics in the 1990s allowed the identification of molecular components related to the two strategies. The gene encoding the $\mathrm{Fe}^{+2}$ transporter related to strategy I, Iron Regulated Transporter 1 (IRT1), was cloned in Arabidopsis thaliana by functional complementation of Saccharomyces cerevisiae mutants defective in iron acquisition (Eide et al., 1996). Other IRT1 homologues were soon identified in yeast showing capacity for zinc transport (Zinc Regulated Transporter, ZRT) (Zhao and Eide, 1996a,b), which led to the ZIP (Z Proteins) denomination of this new protein family (Grotz et al., 1998). Some homologues of ZIP/IRT have already been characterized in Arabidopsis, tomato and soybean (Grotz et al., 1998; Eckhardt et al., 2001; Vert et al., 2001; Moreau et $a l ., 2002)$. The ferric-chelate reductase component related to strategy I was identified in Arabidopsis (AtFRO2) (Robinson et al., 1999) and later in pea (PSFRO1) (Waters et al., 2002). The FRO genes belong to a super family of flavocytochromes located in the cellular membrane that transfer electrons from intracellular donors such as NADPH to extracellular acceptors such as iron or molecular oxygen. Transposon-tagging from a maize mutant defective in iron uptake allowed the cloning of Yellow Stripe 1 (YS1), the only molecular component related to the transport of the $\mathrm{Fe}^{3+}$-phytosiderophore complex characterized to date (Curie et al., 2001).

Additional components related to iron homeostasis have been characterized. Although not related to mineral uptake, these components are probably involved in iron intracellular targeting and storage. Members of the widely distributed NRAMP (Natural Resistance-Associated Macrophage Protein) family of cation transporters have also been characterized in Arabidopsis and rice (Belouchi et al., 1997; Thomine et al., 2000). Experimental evidence for a role of AtNRAMP1, 3 and 4 and OsNRAMP1 in iron homeostasis already exists. AtNRAMP1 may be related to iron subcellular transport and its targeting to storage compartments such as vacuoles or plastids (Curie et al., 2000). Ferritin has a well established role in iron storage and buffering of the mineral availability in the cytoplasm of mammalian cells. In plants, Ferritin is localized in the plastid strome, where it performs a similar function (Ragland et al., 1990). Ferritin cDNAs have been isolated from pea, soybean, maize and Arabidopsis (Briat and Lobréaux, 1997).

Currently, plant genomics allows the fast identification of molecular components related to mineral homeostasis. This is possible through database searches for sequences homologous to proteins already characterized. With this approach, recent reports describe the identification of six genes related to the NRAMP family and fifteen genes related to the ZIP family in Arabidopsis (Mäser et al., 2001), as well as eight ORFs with homology to maize YS1 (Curie et al., 2001).

Once identified, these new components can be molecularly characterized and used for more detailed studies. The analysis of the Arabidopsis EST database and the availability of its complete genome allowed the identification of three new patterns of ferritin, in addition to the already described AtFer1. These new forms provide the first genome-wide study of the expression of ferritin genes in plants (Petit et al., 2001b).

Together with Arabidopsis, rice stands out as a model for plant genomics (Shimamoto and Kyozuka, 2001). The International Rice Genome Sequencing Project (IRGSP), with roots in the Japanese Rice Genome Project, proposes the sequencing of the whole $430 \mathrm{Mb}$ of the rice genome of the japonica variety, cultivar Nipponbare, based on a clone-by-clone approach, aiming at a high accuracy of $10^{-4}$ (Eckardt, 2000; Delseny et al., 2001). In 2001, the IRGSP was highly propelled by the incorporation of the Monsanto rice genome draft and, at the present time, the IRGSP has submitted sequences to GenBank that comprise over $95 \%$ of the genome (http://www.tigr.org/tdb/e2k1/osa1/ BACmapping/description.shtml). Sequencing is still in progress and the announcement of the draft conclusion is expected very soon (Burr, 2002). In parallel, the Beijing Genomics Institute (BGI) and Syngenta have separately published drafts of the rice genome based on a whole shotgun sequencing methodology. The Chinese team obtained a coverage of $92 \%$ of the genome of the indica variety, cultivar 93-11, with a prediction of 46,022 to 56,615 genes (Yu et al., 2002). The Syngenta draft comprises $93 \%$ of the japonica variety genome, with an estimate of 32,000 to 50,000 genes (Goff et al., 2002). 
Using the indica variety genome, the IRGSP data, and the expressed sequence tags (ESTs) deposited in public databanks, we performed an analysis of the rice genome searching for sequences related to the YS, ZIP, FRO, NRAMP and Ferritin protein families. The purpose of this study was to contribute to the understanding of iron homeostasis dynamics in rice (focusing on gene families involved in uptake, intracellular targeting and storage), and to compare the molecular picture of iron nutrition in this model grass species to Arabidopsis thaliana.

\section{Materials and Methods}

Reference proteins of well established molecular function, representing each of the protein families investigated, were chosen as query sequences for searches in the rice (Oryza sativa) genome databases. These reference proteins were ZmYS1 (AAG17016), AtFRO2 (CAA70770), PsFRO1 (AAK95654), AtZIP1 (AAC24197), AtZIP2 (AAC24198), AtIRT1 (AAB01678), AtNRAMP1 (AAF36535), AtNRAMP2 (AAD41078) and ZmFer1 (CAA58146). Searches were made using the TBLASTN tool (Altschul et al., 1997) against the GenBank databases Highthroughput Genomic Sequences (HTG) and Non Redundant (NR), with search specifications for Oryza sativa [Organism]. The BLAST server used was that of the National Center for Biotechnology Information (http://www. ncbi.nlm.nih.gov/BLAST/). The indica variety genome was screened in a specific database offered in the Genomic BLAST pages of the same server (http://www.ncbi.nlm. nih.gov/PMGifs/Genomes/riceWGS.html). As selection criteria of BLAST hits for genomic sequences, a cutoff e-value of e-10 was previously set.

The genomic sequences found were used to predict putative genes contained within them. Whenever possible, genes were predicted on the basis of sequences generated by the IRGSP, since these sequences present a higher degree of accuracy. To that end, a mixed procedure was adopted combining $a b$ initio gene prediction algorithms of genomic sequence alignments with similar sequences from expressed genes (ESTs and cDNAs). The prediction algorithms were GenScan (Burge and Karlin, 1997; http:// genes.mit.edu/GENSCAN.html), GenomeScan (Burge and Karlin, 1997; http://genes.mit.edu/ genomescan.html), FGENESH (Salamov and Solovyev, 2000; http://www. softberry.com/berry.phtml?topic= gfind), GeneMark.hmm (Borodovsky and Lukashin, unpublished; http://opal.biology.gatech.edu/GeneMark/ eukhmm.cgi) and GrailEXP (Xu and Uberbacher, 1997; http://compbio.ornl.gov/ grailexp/).

The similar expressed sequences were found by BLAST searches against EST and NR databases of GenBank, using the genomic sequence as query. The genomic sequences, predicted sequences and expressed gene sequences were then compared by multiple alignments using DIALIGN 2.1 (Morgenstern, 1999; http://bib iserv.techfak.uni-bielefeld.de/cgi-bin/dialign_submit), that allowed the identification of exon and intron positions on the genomic sequences.

Each new predicted CDS served as a query sequence for new BLAST searches, leading to the identification of the largest possible number of related sequences.

The predicted CDS were translated into amino acids and compared to the reference sequences for the searches and to homologous proteins from Arabidopsis. The algorithm of choice for the multiple alignments of protein sequences was ClustalW 1.8 (Thompson et al., 1994), available through the BCM Search Launcher server (http:// searchlauncher.bcm.tmc.edu/multi-align/multi-align.html).

The multiple alignments were edited with the help of GENEDOC (Free Software Foundation, Inc). All of the proteins with greater than $30 \%$ identity, with at least one of the reference proteins used in the searches, were regarded as functionally similar (homologous) to the reference proteins, receiving the same name (Orengo et al., 1994; Rost and Valencia, 1996; Rost, 1999; Todd et al., 2001). Those sequences that did not conform to this criterion were discarded. Only in the case of OsFRO2 the identity degree of $26 \%$ was accepted due to the conserved functional domains between this protein and the reference proteins.

The selected proteins were evaluated for the presence of transmembrane domains, using the SOSUI algorithm (Hirokawa et al., 1998; http://sosui.proteome.bio.tuat.ac. $\mathrm{jp} /$ sosuimenu0E.html). In some cases, the proteins were analyzed in the protein secondary structure databases MetaFam (Silverstein et al., 2001) and Conserved Domain Database (CDD) (Marchler-Bauer et al., 2002) (http:// www.ncbi.nlm.nih.gov/Structure/cdd/cdd.shtml). Protein alignments obtained with Clustal W 1.8 (Thompson et al., 1994) were used as starting points for phylogenetic analysis, which were based on the Parsimony method with the PAUP* software (version 4.0b 10 (PPC/activec; Swofford, 2002). In all cases, 1000 Bootstrap replications tested the tree topology obtained.

Genes were positioned in the chromosomes in agreement with the IRGSP genetic map, available at the site of the Institute for Genomic Reseach, TIGR. Genetic markers were associated to the IRGSP clones using a genetic marker miner located at the TIGR genetic map browser (http:// www.tigr.org/tdb/e2k1/osa1/BACmapping/description.shtml) and also from the TIGR Rice BLAST server (http:// tigrblast.tigr.org/euk-blast/index.cgi?project $=$ osa1) with searches against the genetic marker database.

The EST searches were done both from reference proteins using TBLASTN and from predicted gene sequences, using BLASTN against the EST databases with search specifications for Oryza sativa [Organism]. The e-value cutoff was 0.01 . The ESTs showing less than $5 \%$ of mismatches in an overlap extension of at least 100 nucleotides of BLAST alignment with a predicted CDS from the 
genomic sequence were considered related to a predicted gene in the chromosome.

ESTs D22266 and AU064475 were sequenced in an automatic sequencer MegaBACE DNA Analysis System (Amersham Biosciences) with the M13 -20 and reverse primers.

Sequences and annotations of the new putative genes were submitted to GenBank. Accession numbers of new ferritins are AF519570 (OsFer 1) and AF519571 (OsFer2). Other nucleotide sequence data reported are available in the Third Party Annotation Section of the DDBJ/EMBL/ GenBank databases and their accession numbers are shown in the respective tables.

\section{Results and Discussion}

\section{Genomic overview}

With the availability of the BGI and the IRGSP data in GenBank, it was possible to construct an overview of iron homeostasis in rice. As a starting point, the protein families Yellow Stripe, FRO, ZIP, NRAMP and Ferritin, which have positive molecular implications on iron uptake, intracellular targeting and storage in other plants, were cho- sen for analysis. Taking specific members of these families as query sequences, searches for homologous sequences in the non-redundant (NR), expressed sequence tag (EST) and high throughput genomic sequences (HTG) in GenBank current databases were carried out. Thirty-nine new putative genes were predicted. Together with four previously reported sequences, we analyzed in this study a total of forty-three genes possibly involved with iron homeostasis in rice: eighteen genes related to the Yellow Stripe $\mathrm{Fe}^{3+}$-phytosiderophore transporter, two genes homologous to the $F R O$ family, thirteen sequences related to the ZIP family (including two IRT genes), eight $N R A M P$ putative genes and two ferritin genes. Since the PAC and BAC clones of the IRGSP are linked to genetic markers, it was possible to estimate the relative position of the sequences in the genetic map (Figure 1). The location of five putative new genes still remains unknown, which were predicted from the indica data, whose scaffolds were not anchored to genetic maps.

\section{The yellow stripe family}

A strong indication that the maize YS1 gene encodes the $\mathrm{Fe}^{+3}$-phytosiderophore transporter related to strategy II

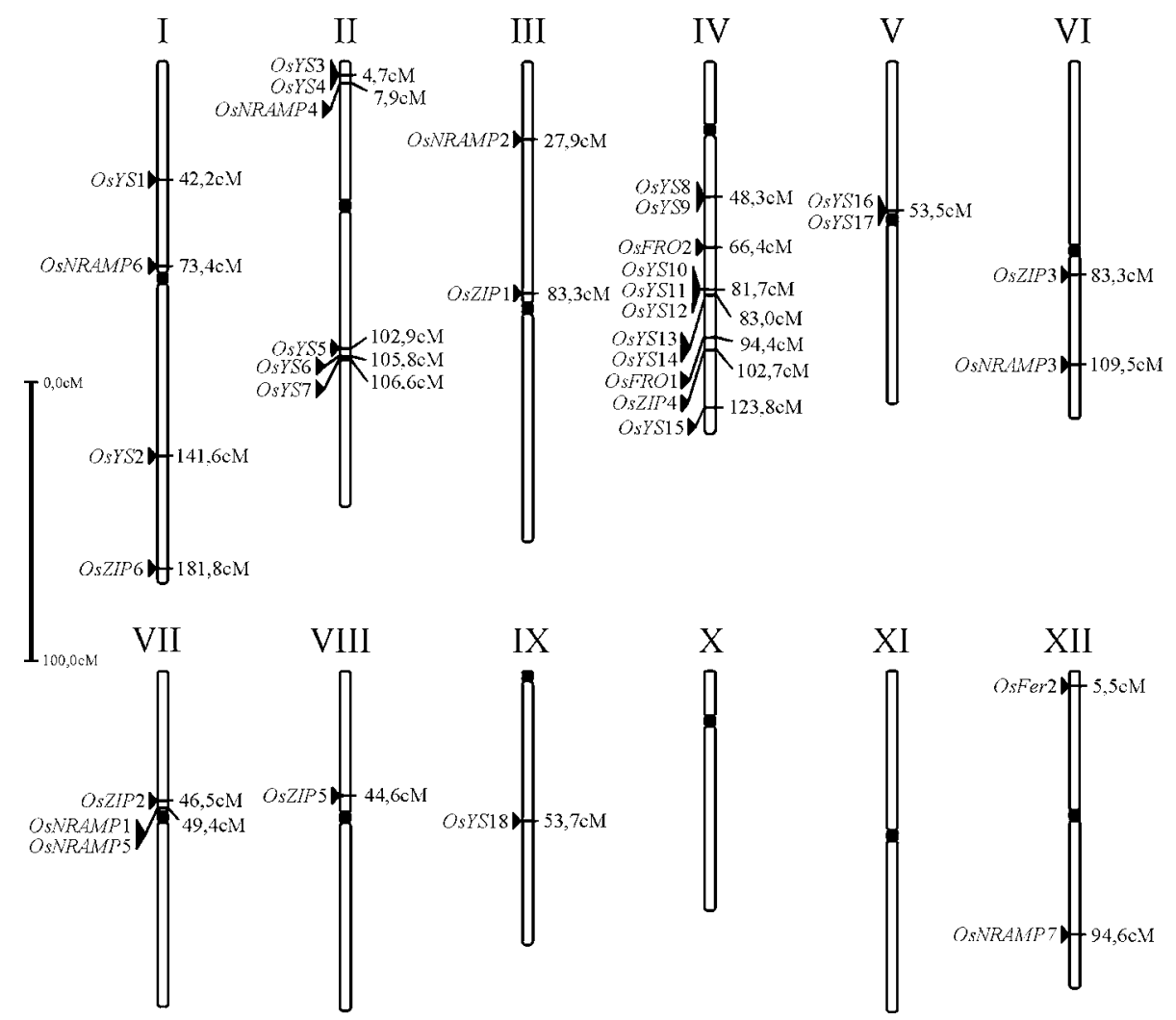

Figure 1 - Chromosome locations of the putative genes related to the iron homeostasis families in rice. All positions in centMorgans (cM) were estimated in accordance to genetic markers assigned to the BAC/PAC clones (as shown by the TIGR genetic map browser: http://www.tigr.org/tdb/e2k1/osal/ BACmapping/description.shtml) related to the predicted sequences. Roman numbers indicate each of the twelve rice chromosomes depicted in white with cetromeres shown in black. The proposed gene nomenclature is as follows: OsYS, Oryza sativa Yellow Stripe-like; OsFRO, Oryza sativa Ferric-chelate reductase-like; OsZIP, Oryza sativa Zip-like; OsNRAMP, Oryza sativa Nramp-like; OsFer, Oryza sativa Ferritin-like. It was not possible to assign a position for five putative genes (OsIRT1, OsIRT2, OsZIP9, OsZIP10, OsZIP11), since these genes were predicted from the BGI scaffolds not anchored to any rice chromosome map. 
found in grass species came from the demonstration of its transport specificity: heterologous expression of a $Y_{s} 1$ clone in a Saccharomyces cerevisiae mutant lineage defective in iron acquisition resulted in functional complementation when $\mathrm{Fe}^{3+}$ was supplied chelated with deoxymugineic acid, but not when Fe-citrate was the mineral source. Expression of Yellow Stripe1 (YSI) in maize roots is increased under mineral deficiency (Curie et al., 2001).

In addition to the eight members of the Yellow Stripe family found in Arabidopsis, there is also a vast number of homologues found in public DNA sequence databanks, related to expressed genes in other plants. In a broader sense, the YS1 protein also belongs to a superfamily of oligopeptide transporters, called OPT (Oligopeptide Transporter) (Yen et al., 2001). The OPT family is particularly well characterized in yeast (Hauser et al., 2001) and Arabidopsis (Koh et al., 2002), in which its members function in the acquisition of oligopeptides (tetra and pentapeptides) from the environment. A wider role for this protein family in the transport of nicotianamine (NA) in plants has also been suggested (Curie et al., 2001).

Through searches for homologous sequences related to the maize transporter YS1, we found several orthologs in the rice genome, with at least forty six related ESTs. All of these correspond to eighteen genomic sequences related to the IRGSP BAC/PAC clones. Only OSYS1 had already been described and had CDS (coding DNA sequence) prediction submitted to the PLN subdivision of GenBank (for plant, fungal and algal sequences), which required the coding regions to be established for the other sequences. They were predicted from the respective IRGSP clones. The patterns of exons are shown in Table 1, with the numerals corresponding to the positions of the coding regions relative to the genomic clones. We propose a nomenclature for the maize YS1 orthologs in rice as "Oryza sativa Yellow Stripe-like" $(O s Y S)$, numbered 1 to 18 as the order of appearance in the genome (Figure 1, Table 1). In four cases there are $O s Y S$ genes positioned in tandem in the same genomic locus, evidence of recent gene duplications leading to the expansion of this family. The CDS length of the new sequences is around 2000 nucleotides, which is similar to the 2049 found in maize YSI (Curie et al., 2001). Most of the sequences have a structure of six to eight exons, which is in good agreement with the seven exons found in maize YS1. There are related ESTs for ten out of eighteen YS1 rice orthologs, indicating that these are functional genes. For the putative CDS without related ESTs, further studies will be required to attest their functionality.

The seventeen newly identified genes and the previously reported $O s Y S 1$ were translated into amino acids and, together with maize YS1, analyzed in the secondary protein database MetaFam. In all cases, hits occurred with the profile of the OPT family. For maize YS1 protein, the obtained e-value related to the OPT family profile was $2.7 \mathrm{e}-98$, con- firming that the Yellow Stripe family belongs to the OPT super family of oligopeptide transporters (Yen et al., 2001). Similar e-values were found for the rice sequences. The similarity with the OPT family also seems to be functional, since maize YS1 transports compounds derived from mugineic acid, a non-protein amino acid, structurally similar to small peptides. The eighteen putative OsYS proteins, maize YS1 and the eight orthologs found in Arabidopsis were compared at protein level based on multiple alignments. All the putative OsYS proteins showed more than $30 \%$ identity with maize YS1. The most similar proteins were OsYS7 with 75\% identity and OsYS6, 13 and 14 with more than $60 \%$ identity with the maize transporter. The rice YS members also share a high level of similarity with the AtYS-like proteins, with more than $40 \%$ identity in most cases. The presence of transmembrane domains was also investigated for the eighteen OsYS proteins. The result was somewhat heterogeneous with the majority of proteins presenting 12 to 14 transmembrane domains (Table 1), close to the 12 observed in the maize YS1 (Curie et al., 2001).

A better visualization of the relationships between YS protein sequences was achieved by a phylogenetic analysis. Besides the YS members of rice, Arabidopsis and the maize YS1, the OPT family members from Arabidopsis (AtOpt1 and AtOpt5) were also included in the analysis (Koh et al., 2002), as well as two sequences from the Fungi kingdom, Candida albicans CaOpt1 (Lubkowitz et al., 1997) and $S$. cerevisiae ScOpt1 (Hauser et al., 2000). The tree shown in Figure 2 was obtained after 6603 steps of a heuristic search using branch-swapping options. The Consistency Index (CI) was 0,6441 . The YS homologues were clustered separately from the representatives of the OPT family. OsYS16 and OsYS17 figured at the base of the YS branch, followed by OsYS1, showing that these proteins were more divergent from maize YS1 and the other YS members were more closely clustered. These results indicated that these new putative genes found in rice are homologous to the maize YS and probably have a similar transporter function.

\section{The ferric reductase oxidase (FRO) family}

In strategy I plants, the ability to change the redox state of $\mathrm{Fe}^{3+}$ in the soil was early recognized as an obligatory step for iron uptake (Chaney et al., 1972). The ferric-chelate reducing activity of yeast cells provided the first insight into the molecular basis of this physiological function. The FRE1 gene was isolated based on complementation of the $f r d-1$ yeast mutant, defective in the ferric-chelate reductase activity (Dancis et al., 1992).

By means of PCR screenings of cDNA and genomic libraries using degenerate primers specially designed for the conserved cofactor binding sites from yeast and human sequences, it was possible to isolate the AtFRO1, AtFRO2 and AtFROHC genes from Arabidopsis (Robinson et al., 1997; Robinson et al., 1999) and PsFRO1 from pea (Pisum Sativum) (Waters et al., 2002). The genomic location of 


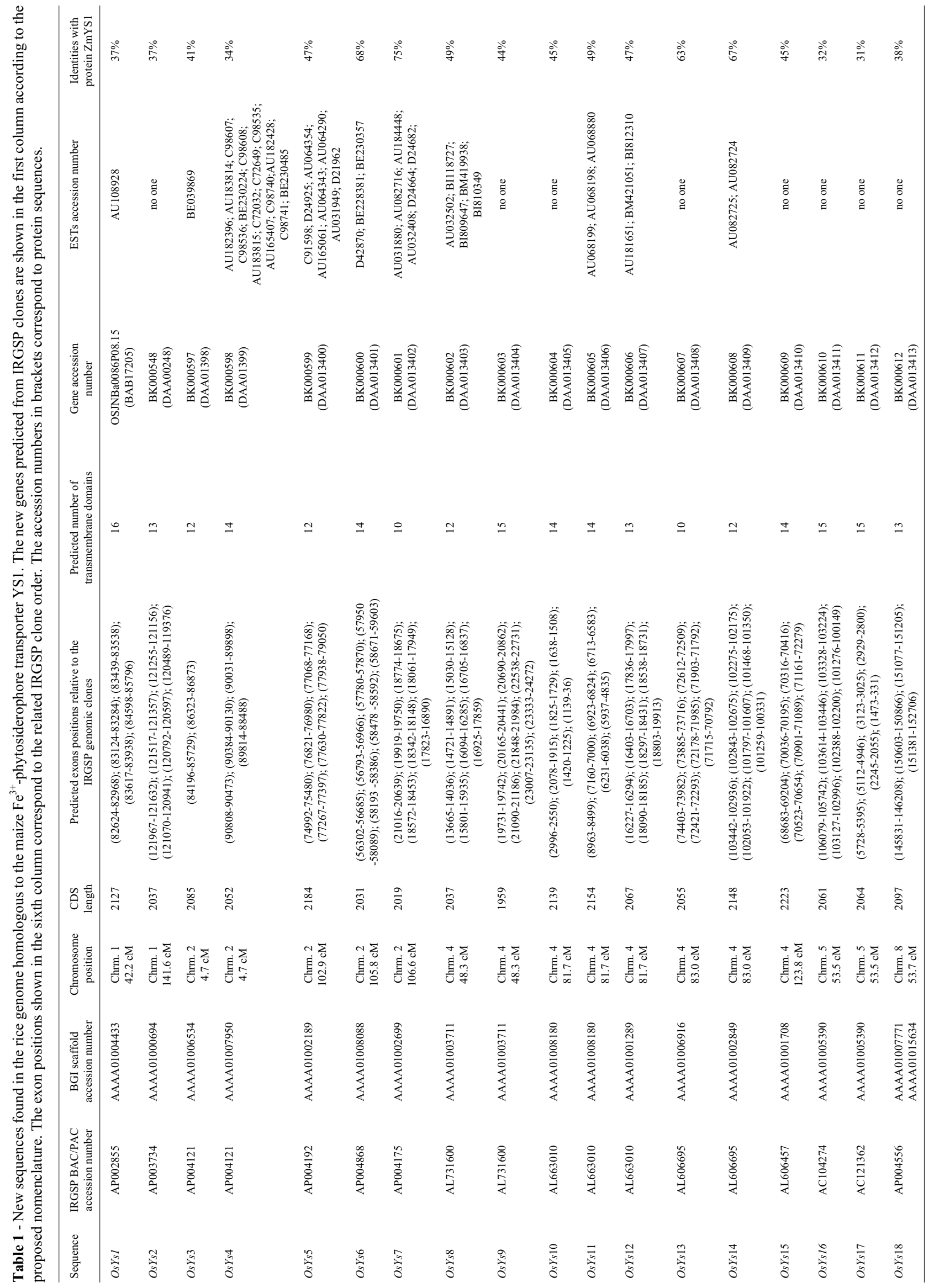




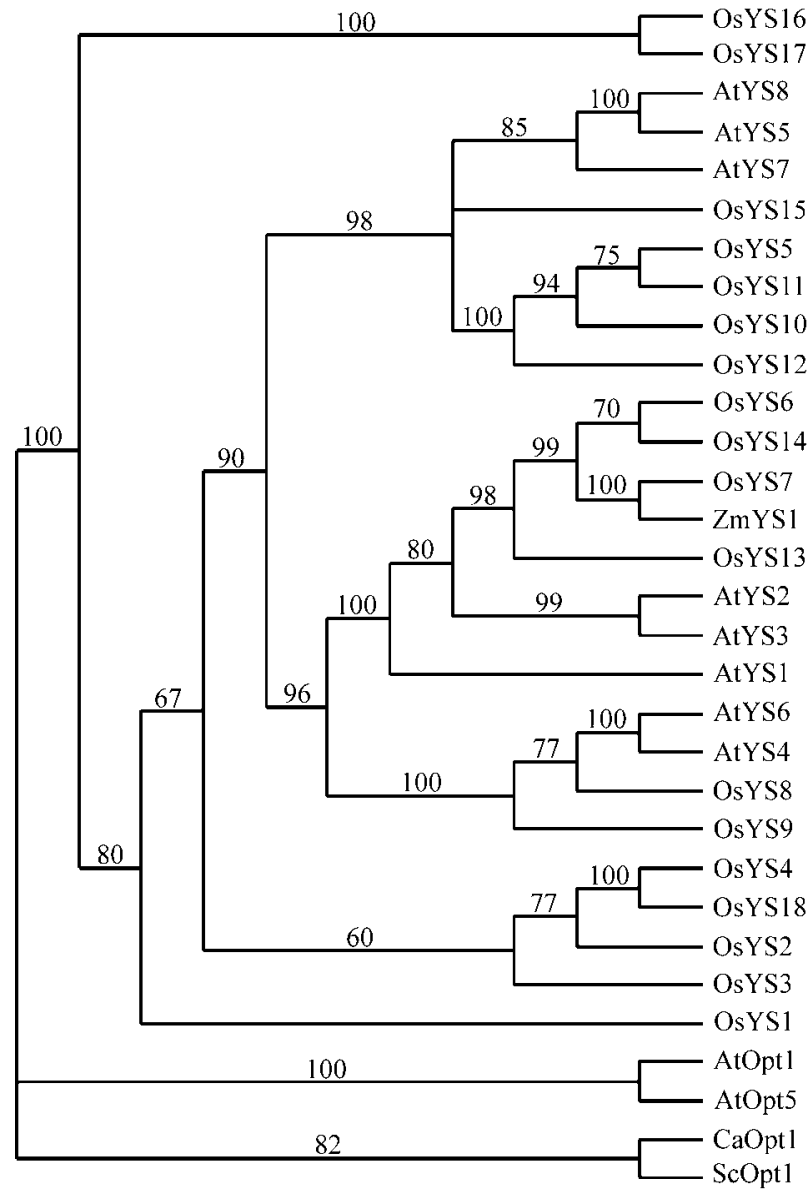

Figure 2 - Phylogenetic analysis of the Yellow Stripe family using PAUP (Swofford, 2002) after alignment of the sequences with Clustal W (Thompson et al., 1994). The resulting tree shows the relationships between the new 18 members predicted from the rice genome (OsYS) and the eight representatives found in Arabidopsis (AtYS) and the Zea mays YS1 (ZmYS1). Opt members from Arabidopsis (AtOpt), Candida albicans (CaOpt1) and $S$. cerevisiae (ScOpt1) were joined together as an outgroup. Accession numbers of nucleotide sequences and corresponding predicted OsYS proteins are shown in Table 1. Accession numbers to the other proteins are as follows: ZmYS1: AAG17016; AtYS1: NP 567694; AtYS2: NP_197826; AtYS3: NP_200167; AtYS4: NP_198916; AtYS5: NP 566584; AtYS6: NP 566806; AtYS7: NP 176750; AtYS8: NP 564525; AtOpt1: BAB08658; AtOpt5: CAB43855; CaOpt1: AAB69628; ScOpt1: NP_012323. Values associated with tree branches indicate the number of times (in percent) that each branch topology was found during bootstrap analysis, with 1000 replications.

AtFRO2 proved to be allelic to the ferric-chelate reductase impaired mutation, that could be restored with the ectopic expression of AtFRO2 (Robinson et al., 1999). PsFRO1 also demonstrated ferric ion-chelating activity in assays using PSFRO1-expressing yeast cells (Waters et al., 2002). The expression patterns of AtFRO2, AtFROHC and PSFRO1 in roots were increased under limiting iron availability. The analysis of predicted proteins from these new genes puts them in the same superfamily of flavocytochromes, to which belong the yeast FRE1 protein and a subunit of the respiratory burst oxidase (gp91-phox) from human neutrophyls (Robinson et al., 1997; Robinson et al.,
1999; Waters et al., 2002). Additional homologues have been detected in Arabidopsis and rice which could presumably act as burst oxidases generating reactive oxygen species as a defense against pathogens (Groom et al., 1996; Torres et al., 1998). A common feature of this superfamily is the high similarity around the FAD, NADPH and heme binding sites, suggesting catalytic similarity between FRO and mammalian proteins. The main difference is that in the FRO members $\mathrm{Fe}^{3+}$ acts as the final acceptor for the transferred electron instead of molecular oxygen, as in the case of burst oxidases (Robinson et al., 1999).

We have searched for homologous genes in the genomic and expressed rice sequences using the PsFRO1 and AtFRO2 proteins as query sequences. Only two genomic sequence patterns with significant BLAST hits were found in the IRGSP clones and BGI scaffolds. The predicted CDS showed related proteins with lengths and structures similar to the query sequences and identities higher than $25 \%$ with the reference proteins. The new CDS were named Oryza sativa FRO1 (OsFRO1) and OsFRO2 (Table 2). OSFRO2 is a functional gene since there are four identical rice ESTs found in the expressed gene databases. There is only one wheat cDNA with high similarity for OSFRO1 (529 identical nucleotides of 538 as shown by BLAST alignment), derived from a substracted cDNA library of cold -stressed seedlings. Excluding this cDNA and a few other ESTs from wheat, it is interesting to note that most expressed genes similar to OsFRO1 found in databases are from non-graminaceous plants.

The derived OsFRO proteins were analyzed for putative transmembrane domains. OsFRO1 contains 10 transmembrane spanning regions whereas OsFRO2 has 11. These proteins were also searched against the Conserved Domain Database (CDD), presenting significant hits only with the profile of Ferric reductase transmembrane family, to which gp91-phox and FRE1 from yeast and the Arabidopsis and pea FRO proteins belong. In order to examine with more attention the similarities of the FRO family proteins, we performed a multiple alignment of the predicted OsFRO polypeptides with the previously described FRO members from Arabidopsis and pea, also including AtRbohF, a respiratory burst oxidase member from Arabidopsis (Figure 3). The OsFRO proteins have all the expected sites for heme and dinucleotide cofactor binding, suggesting a similar functional activity for these new enzymes. OsFRO1 has greater than $39 \%$ identity with the reference proteins. OsFRO2 showed only $26 \%$ identity with AtFRO2 and PsFRO1, within a range (20-30\% identity) for which it is difficult to infer homology, the so-called "twilight zone" (Rost and Valencia, 1996). However, considering the highly conserved amino acids around the catalytic sites (Figure 3), it is reasonable to regard OsFRO2 as functionally related to other FRO proteins.

A dendrogram was constructed based on a multiple alignment including the previously compared sequences, 


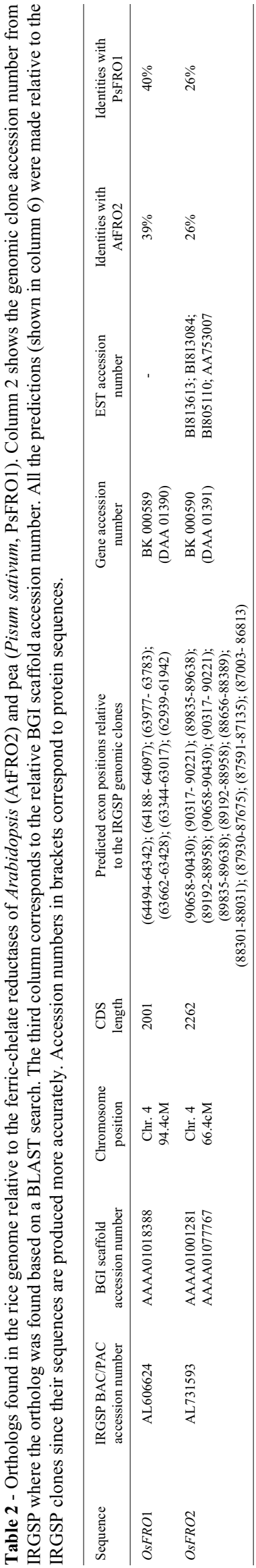

six Arabidopsis FRO relatives found in the GenBank non redundant database and another burst oxidase sequence from rice (Figure 4). The resulting tree was obtained after 3720 steps of a branch and bound search. The Consistency Index was 0,8798 . The tree topology clearly puts the burst oxidases in a separate clade from the FRO sequences. There is also a subdivision in the FRO family in two separate groups. OsFRO1 was clustered in the group comprising the AtFRO1, 2 and $\mathrm{C}$ and the PsFRO1, indicating that this rice protein is closely related to the previously described members of the FRO family. OsFRO2 was grouped together with three Arabidopsis proteins that present a lower degree of similarity with AtFRO2 and PsFRO1 (nearly 25\% identity and $45 \%$ similarity) but are clearly distinct from the burst oxidases members.

\section{The ZIP family}

Members of the ZIP family are amongst the most studied metal transporters in plants (Guerinot, 2000; Mäser et al., 2001). Arabidopsis IRT1 is the best characterized so far. Functional complementation assays of yeast mutants clearly established a preference for the transport of $\mathrm{Fe}^{2+}$ instead of $\mathrm{Fe}^{3+}$ (Eide et al., 1996). Moreover, IRT1 is expressed in roots under iron starvation and the corresponding gene product only accumulates under the same circumstances, even when the gene is overexpressed by means of a $35 \mathrm{~S}$ promoter construct. This pattern suggests control at the protein stability level (Connolly et al., 2002). Reporter gene expression and in situ hybridization of the IRT1 protein showed its localization at the plasma membrane of root epidermal cells. These results, combined with the fact that Arabidopsis IRT1 knock out mutants are lethal, are strong evidence that this protein is the main component responsible for iron uptake from soil in limiting conditions of this mineral (Vert et al., 2002). IRT2 from Arabidopsis is also expressed during iron starvation in root epidermal cells (Vert et al., 2001).

There is molecular evidence for a function in cation transport for Arabidopsis ZIP1, ZIP2 and ZIP3, as they could restore zinc uptake in the yeast double mutant $z r t 1$ and zrt2. Northern blot experiments showed that ZIPI and ZIP3 are expressed in roots under zinc deficiency, while ZIP4 is expressed both in roots and shoots under the same condition. Therefore, these ZIP members have been implicated in $\mathrm{Zn}^{2+}$ uptake from the soil (Grotz et al., 1998). A feature observed for some members of this family is a broader affinity for cation transport. The Arabidopsis ZIP members are currently being investigated by reverse genetic methods, which will provide further information about the molecular functions of these genes (Mäser et al., 2001).

The IRT1, ZIP1 and ZIP2 Arabidopsis proteins were used as query sequences to search for ZIP family homologues in rice. Several sequences were found, corresponding to thirteen putative predicted genes (Table 3). The 


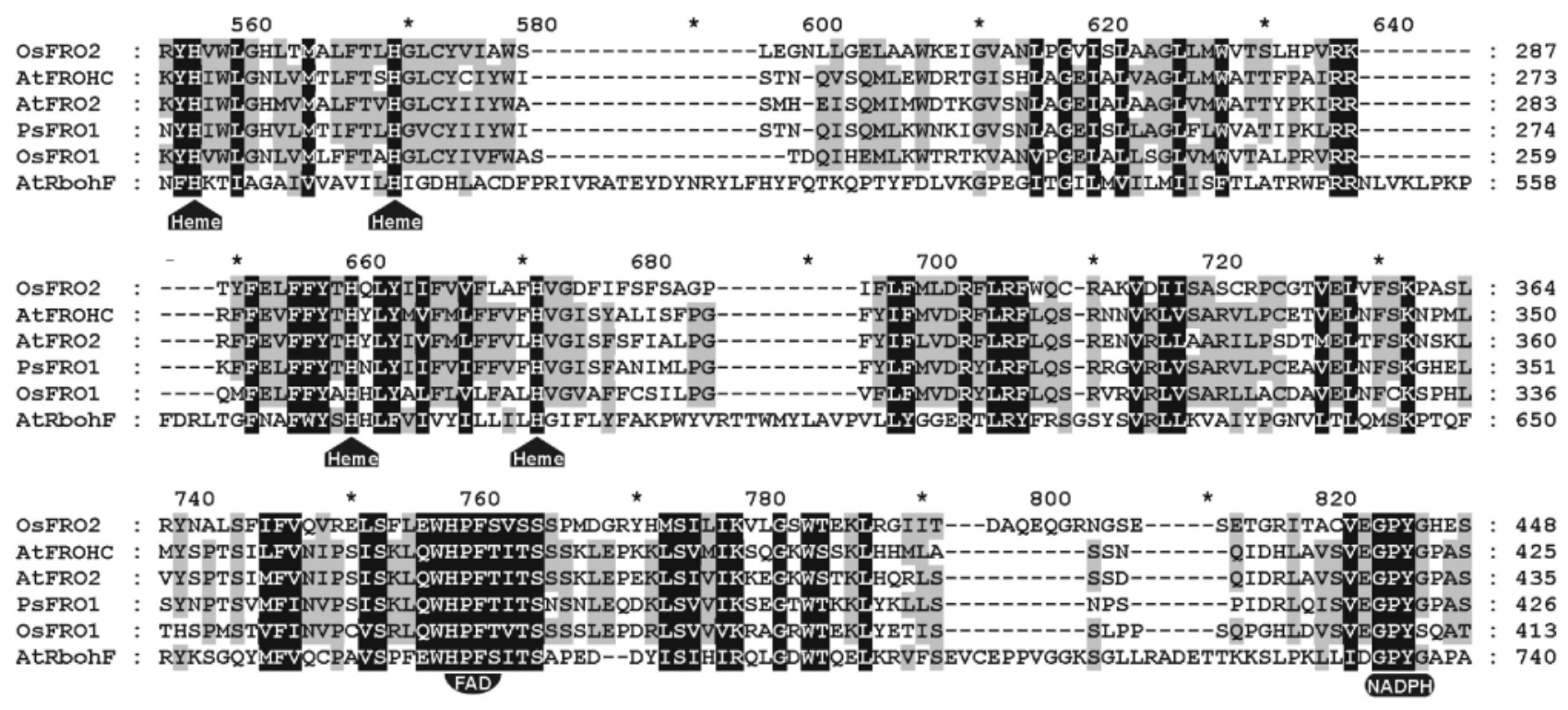

Figure 3 - Conservation of amino acids around the prosthetic binding sites in the FRO family. The multiple alignment was performed using ClustalW algorithm. Only the central parts of the proteins are shown. The four heme-coordinating histidine $(\mathrm{H})$ residues and the dinucleotide binding motifs (FAD, HPFT and NAPH, GPYG) are indicated under the block of alignments in the respective positions. Accession numbers of nucleotide sequences and corresponding predicted OsFRO proteins are shown in Table 2. Accession numbers to the other proteins are as follows: AtFrohC: AAB72168; AtFRO2: CAA70770; PsFRO1: AAK95654; AtRbohF (Arabidopsis thaliana Respiratory burst oxidase F): BAA28953. Black color indicates regions in the alignment with $100 \%$ similarity and gray color indicates regions with at least $60 \%$ similarity.

previously described gene OSIRT1 finds matching sequences in the BGI scaffolds AAAA01002856 and AAAA01010833. A gene product presenting 77\% identity with OsIRT1 and 49\% identity with AtIRT1 was predicted based on the BGI AAAA01010833 scaffold. Because of its high similarity with OSIRT1, this gene was named OSIRT2. The other eleven predicted genes, due to their greater similarity to AtZIP1 and AtZIP2, were named OsZIP1 to OsZIP11. Most CDS were predicted from clones sequenced by the IRGSP, whereas OsZIPO to OsZIPI1 and OSIRT1 and OsIRT2 were only found in BGI scaffolds. CDS OsZIP9, OsZIP10 and OsZIP11 are incomplete, since the corresponding scaffolds are of short extension. Sixteen ESTs corresponding to six of these predicted CDS confirm that at least these six genes are functional (Table 3). The fact that $O s I R T 1$ and $O s Z I P 9$ to $O s Z I P 11$ were exclusively predicted from genomic sequences corresponding to BGI scaffolds suggests that these could be genes restricted to the indica variety.

The ESTs related to the $O S I R T 2$ gene were generated from cDNAs derived from both the japonica variety, cultivar Nipponbare, and the indica variety, cultivar Pokkali. The same was observed for the other genes with ESTs that correspond to cDNAs derived from different varieties (Table 3). Therefore, the greater number of genomic sequences related to the indica variety may reflect the higher genomic coverage level of the BGI libraries.

The predicted gene sequences were translated into amino acids and compared both by multiple alignment and phylogenetic analysis to the Arabidopsis ZIP proteins and yeast ZRT1 and ZRT2. Four trees were obtained after 4058 steps of a heuristic search with branch and bound options. A strict consensual method generated the tree depicted in Figure 5. The Consistency Index was 0.6353 . The tree topology was tested with 1000 Bootstrap replications. The proteins resulting from the new rice sequences showed an extension from 350 to 390 amino acid residues, with a similarity among themselves ranging from 33 to $77 \%$ and more than $35 \%$ identity to at least one of the three reference proteins used in the searches (Table 3). All these proteins are grouped in the phylogenetic tree together with the Arabidopsis ZIP polypeptides in a superclade that separated them from the yeast ZRT proteins. OsZIP1 and OsZIP6 are more distantly related to the other ZIP proteins, being grouped with AtZIP2 and AtZIP11, closer to the root of the ZIP family.

Transmembrane domains were predicted for the OsIRT1, OsIRT2 and OsZIP1 to OsZIP8 proteins, leaving out the incomplete polypeptides OsZIP9, 10 and 11. Seven to nine transmembrane domains were found (Table 3), in good agreement with the eight regions observed for the Arabidopsis ZIP family members (Mäser et al., 2001). Also in line with the Arabidopsis ZIP proteins, a central portion with a low degree of conservation, the so called variable region (Guerinot, 2000), was also present in the rice ZIP proteins. This region contained, in the majority of examined sequences, several histidine residues, presumably metal binding sites. Three other histidine residues, located in the II, IV and V transmembrane domains, are conserved in all rice ZIP sequences. Point mutations in these residues of the 


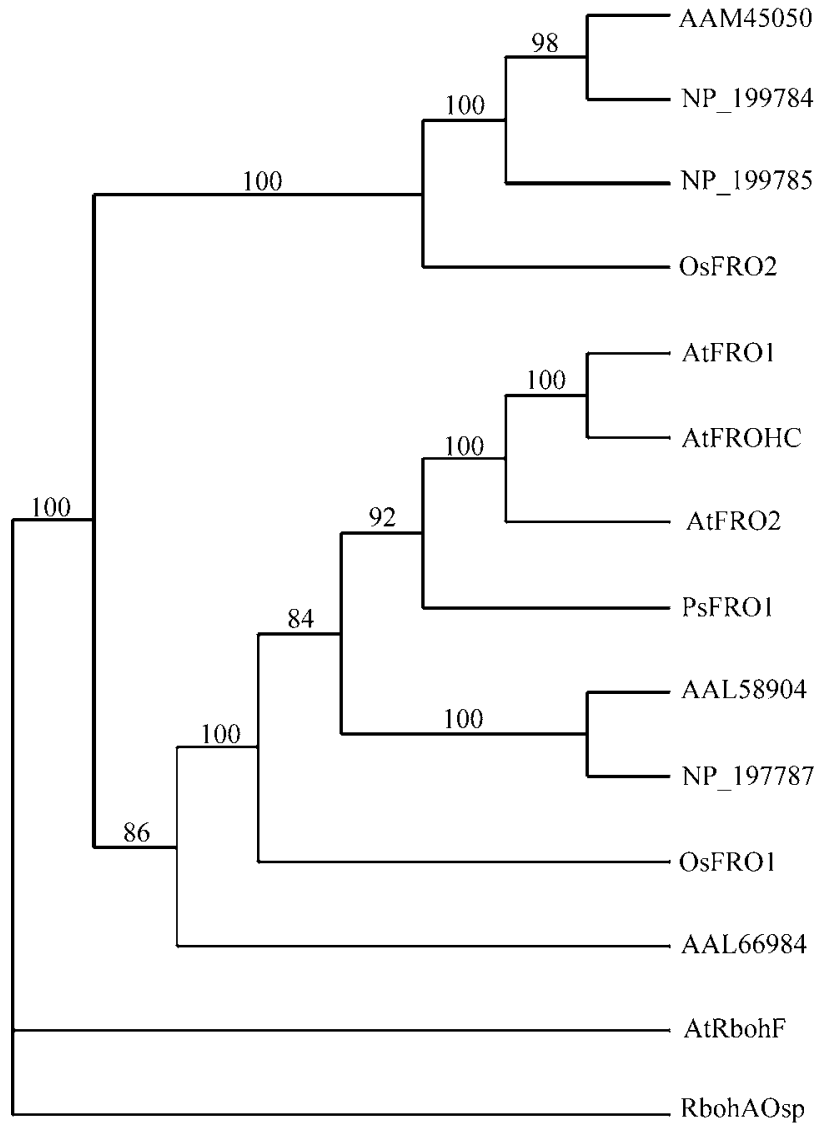

Figure 4 - Phylogenic analysis of the FRO protein family. Accession numbers of nucleotide sequences and corresponding predicted OsFRO proteins are shown in Table 2. Accession numbers to the other proteins are as follows: AtFRO1: CAA70769; AtFRO2: CAA70770; AtFrohC: AAB72168; PsFRO1: AAK95654; AtRbohF (Arabidopsis thaliana Respiratory burst oxidase F): BAA28953; RbohAOsp (Oryza sativa respiratory burst oxidase A): AAB87790. The six additional Arabidopsis paralogs are discriminated in the dendrogram with the respective accession numbers.

Arabidopsis IRT1 abolished the protein capacity to transport iron and other minerals (Rogers et al., 2000). The conservation of these same residues in the rice ZIP proteins suggests an important catalytic function kept unaltered in the large gene family.

\section{The NRAMP family}

The first Nramp (Natural Resistance-Associated Macrophage Protein) gene to be identified was the mammalian Nrampl, which encodes a macrophage membrane protein believed to control divalent cation concentrations within the phagosome, and, by this means, to regulate replication of engulfed bacteria (Williams et al., 2000). The NRAMP protein family, with members also in Drosophila melanogaster, Saccharomyces cerevisiae and rice (OsNRAMP1), was defined by a highly conserved hydrophobic core with 10 transmembrane domains, several invariant charged residues and other features typical of transporters or channels (Cellier et al., 1995).

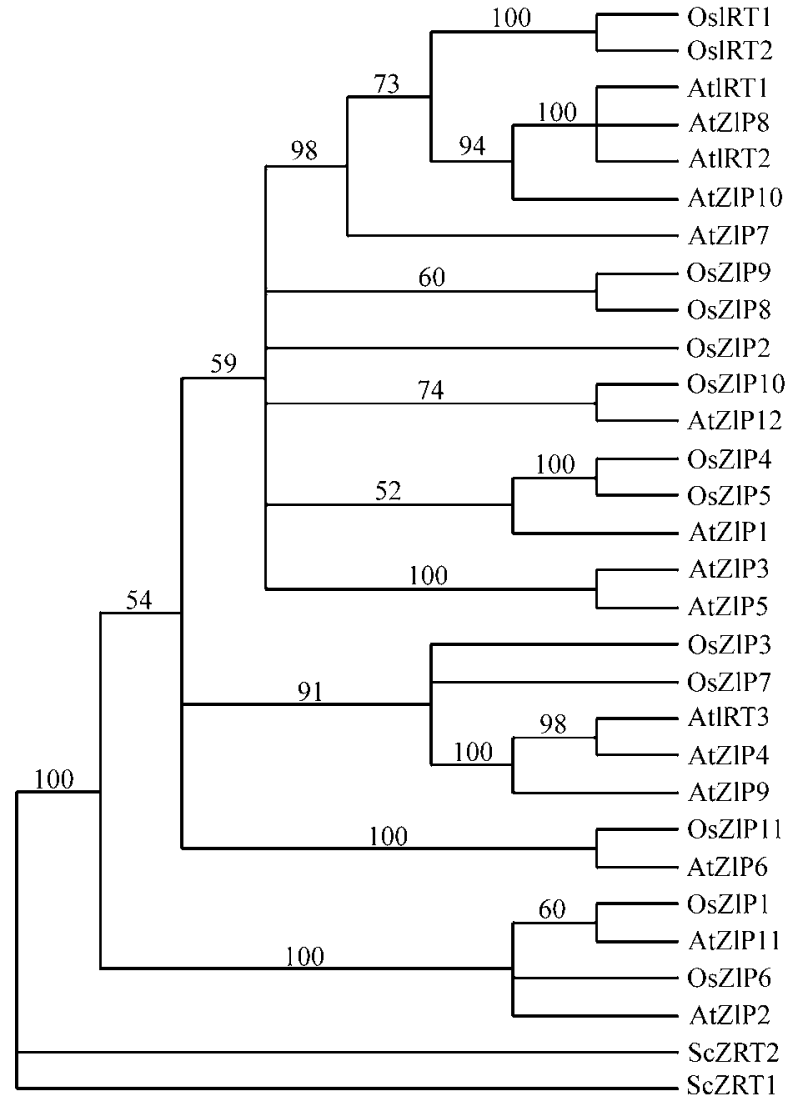

Figure 5 - Phylogenetic analysis of the ZIP protein family from Oryza sativa and Arabidopsis with Zinc regulated transporters ZRT1 and two from $S$. cerevisiae as outgroup. Accession numbers of nucleotide sequences and corresponding predicted OsZIP proteins are shown in Table 3. Accession numbers to the other proteins are as follows: AtIRT1: AAB01678; AtIRT2: NP_193703; AtIRT3: NP 564766; AtZIP1: AAC24197; AtZIP2: AAC24198; AtZIP3: AAC24199; AtZIP4: NP 172566; AtZIP5: NP 172022; AtZIP6: NP 180569; AtZIP7: AAL38434; AtZIP8: AAL83293; AtZIP9: NP_195028; AtZIP10: NP_174411; AtZIP11: NP_564703; AtZIP12: NP_201022; ScZRT1: P32804; ScZRT2: NP_013231.

Two groups presented evidence for the involvement of plant NRAMP proteins in iron transport. Curie et al. (2000) showed that both rice and Arabidopsis NRAMP1 were able to complement the fet3fet 4 yeast mutant, defective in low- and high-affinity iron transport, with the Arabidopsis protein being more efficient than the rice one. They also showed that AtNrampl transcript accumulated in response to iron deficiency in roots. Furthermore, overexpression of AtNRAMP1 in Arabidopsis increased its resistance to toxic iron concentrations. Thomine at al. showed that AtNramp 3 and AtNramp 4 were more efficient than AtNramp1 in complementing the iron uptake yeast mutant fet3fet 4 . Iron starvation resulted in up-regulation of expression of the Arabidopsis Nramp genes. Disruption of AtNramp3 led to increased cadmium resistance and overexpression of the same gene led to cadmium hypersensitivity. It was suggested that the AtNRAMP3 protein may be involved in iron uptake in roots, with its contribution rel- 


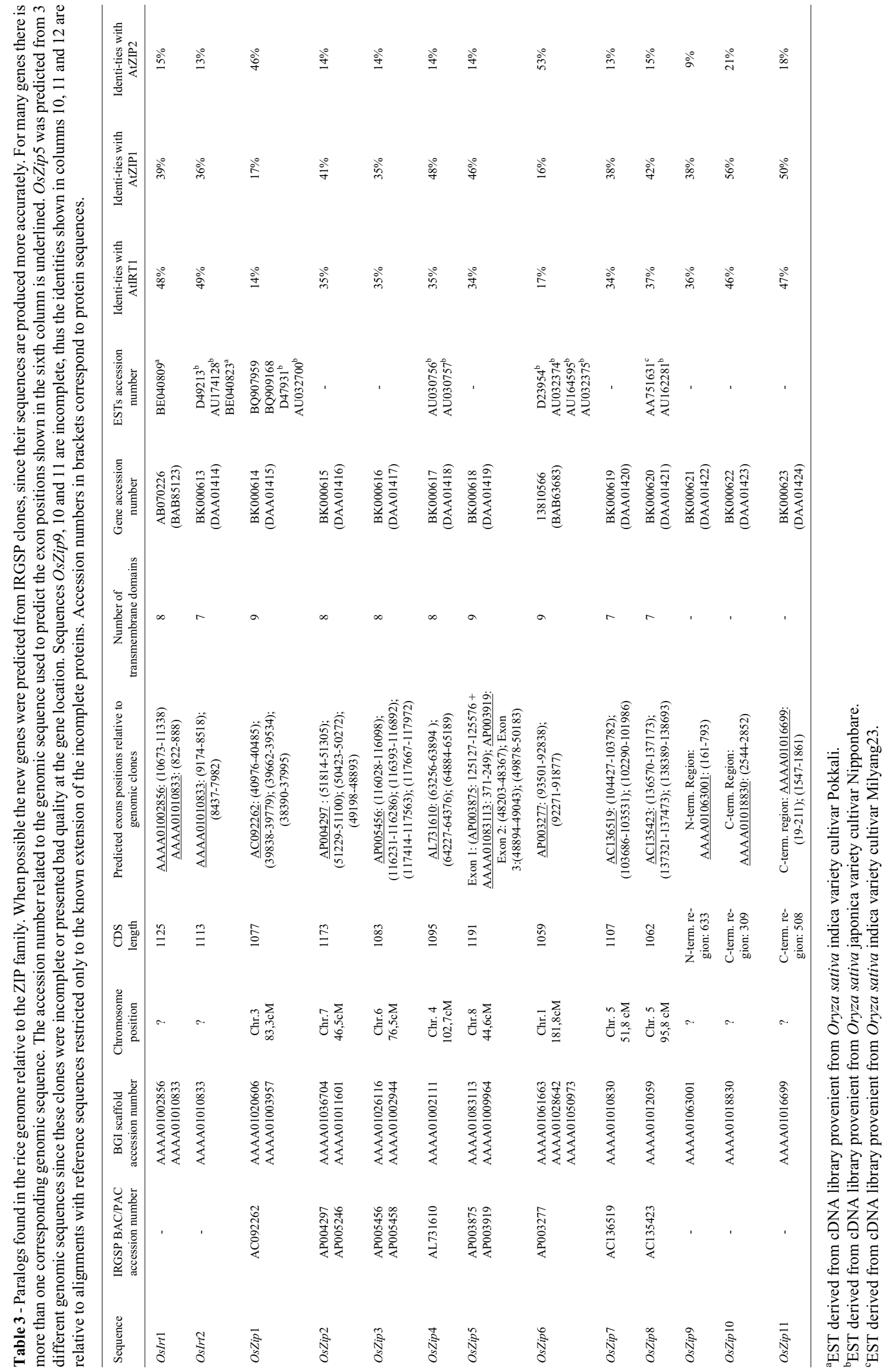


ative to IRT1 being dependent on $\mathrm{pH}$ (Thomine et al., 2000).

The sequences of the Arabidopsis NRAMP1 and NRAMP2 proteins were used as queries to search for NRAMP orthologs in rice. Besides the three NRAMP genes previously known in rice (Belouchi et al., 1997), five new members of this family were predicted based on BGI and IRSGP genomic sequences. These new putative genes were called OsNRAMP4 to OsNRAMP8 (Table 4). The previously deposited (U60767) CDS OsNRAMP3 was incomplete, so we used the predicted complete CDS, based on the IRGSP genomic sequence AP004989, in our analyses. OSNRAMP7 was annotated on the basis of two genomic clones, since the AL772426 sequence has a gap in the region corresponding to the 5'end of the gene. The complete CDS was obtained using scaffold AAAA01007693, that provided the missing region. The OSNRAMP sequences were approximately $1500 \mathrm{bp}$ long. The corresponding genes had 12 exons in OsNRAMP6 and 13 exons in OsNRAMP1, 3, 4 and 5 . In contrast, OsNRAMP 8 had only 3 exons and $O S N R A M P 2$ and 7 had 4 exons. There were nineteen ESTs related to OSNRAMP1, 2, 5, 6 and 7, indicating that these are expressed genes. For the other predicted genes, molecular evidence needs to be acquired in order to fully establish their functionality. The two NRAMP members with the largest number of related ESTs, OSNRAMP1 and OSNRAMP5, were found in the same chromosomal locus, suggesting a recent event of gene duplication.

The predicted CDS for the eight OSNRAMP genes were translated into amino acids and analyzed in multiple alignment comparatively to the Arabidopsis NRAMP. All gene products showed over $35 \%$ identity with the reference proteins (Table 4). OsNRAMP2, 7 and 8 had higher similarity with AtNRAMP2, displaying 60 to $66 \%$ identity with this protein. The other polypeptides were more closely related to AtNRAMP1, reaching up to $68 \%$ identity in the case of OsNRAMP3. Also, the OsNRAMP products were highly conserved when compared among themselves, showing up to $72 \%$ identity and $81 \%$ similarity, as in the case of OsNRAMP7 and OsNRAMP8. All of the OsNRAMP proteins were highly conserved in the region referring to the Consensus Transport Motif (CTM), located between two transmembrane regions in the carboxyterminal region of the proteins. This motif was first identified in bacteria and is present in all Arabidopsis NRAMP proteins (Curie et al., 2000). The transmembrane domain prediction showed that most of the proteins had 10 of these domains, two of them had 11 transmembrane domains and one had only 8 (Table 4). The phylogenetic analysis of protein sequences of the rice NRAMP, Arabidopsis NRAMP and SMF proteins of Saccharomyces cerevisiae is shown in Figure 6. The depicted tree was obtained after 2765 steps of a heuristic search with branch-swapping options. The Consistency Index was 0.8119 . The resulting topology clearly placed the NRAMP family in a different branch from the yeast SMF proteins. The NRAMP family was arranged in 2 separated clades. One grouped the proteins similar to AtNRAMP1 and the other involved those related to AtNRAMP2. In fact, the previously known Arabidopsis and rice NRAMP proteins seem to belong to two subclasses, one including AtNRMP1 and 6 and OsNRAMP1 and 3 and the other including AtNRAMP2 to 5 and OsNRAMP2 (Curie et al., 2000; Maser et al., 2001). In the present study, a correlation was apparent between the number of exons of OSNRAMP genes and the observed branching of the NRAMP protein phylogenetic tree. The proteins with less exons present in their genomic sequences, OsNRAMP2 (4 exons) and OsNRAMP7 and 8 (3 exons each), were grouped in the same branch with AtNRAMP2. The NRAMP proteins with corresponding genomic sequences having a larger number of exons, OsNRAMP6 (12 exons) and OsNRAMP1, 3, 4 and 5 (13 exons each) were in a different branch, clustered with AtNRAMP1.

\section{The ferritin family}

Ferritins are iron storage proteins with highly conserved structure among plants, animals and bacteria. The protein has 24 subunits organized in a spherical shell, and can store up to 4500 iron atoms in its central cavity (Briat and Lobréaux, 1997). In mammals, two different types of ferritin subunits are assigned different functions: the $\mathrm{H}$ subunit contains a ferroxidase site responsible for rapid $\mathrm{Fe}^{2+}$ oxidation, leading to rapid iron uptake to inside the protein, and the L subunit lacks this site but is rich in E residues facing the central cavity of the protein, allowing better nucleation of $\mathrm{Fe}^{3+}$ for long-term storage (Petit et al., 2001b and references therein). Plant ferritins combine both functions in their subunits, which share higher homology with animal ferritins than with bacterioferritins, although they are localized in the plastids, a typical prokaryotic environment (Briat and Lobréaux, 1998).

Plant ferritin genes do not contain IREs (Iron Regulatory Elements), conserved regulatory sequences responsible for the translational derepression of animal ferritin mRNA in response to iron overload (Petit et al., 2001b and references therein). Instead, in the promoters of maize and Arabidopsis ferritin genes, the presence of Iron-Dependent Regulatory Sequences (IDRS), responsible for repression of transcription under low iron supply, was recently shown (Petit et al., 2001a). In maize, regulation of ZmFer 1 by iron is ABA-independent and requires the IDRS, while induction of its expression by $\mathrm{H}_{2} \mathrm{O}_{2}$ does not require a functional IDRS. One form of maize ferritin ( $\mathrm{ZmFer2}$ ) is expressed in shoots in response to ABA treatment, as well as one Arabidopsis ferritin (AtFer2), which is expressed in seeds (Fobis-Loisy et al., 1995; Petit et al., 2001b). AtFerl, 3 and 4 are expressed in response to iron overload, although with different kinetics in the latter. AtFerl and, to a lesser extent, AtFer 3 , are also expressed in response to $\mathrm{H}_{2} \mathrm{O}_{2}$. Both AtFer 3 and AtFer 4 promoters have partly conserved IDRS 


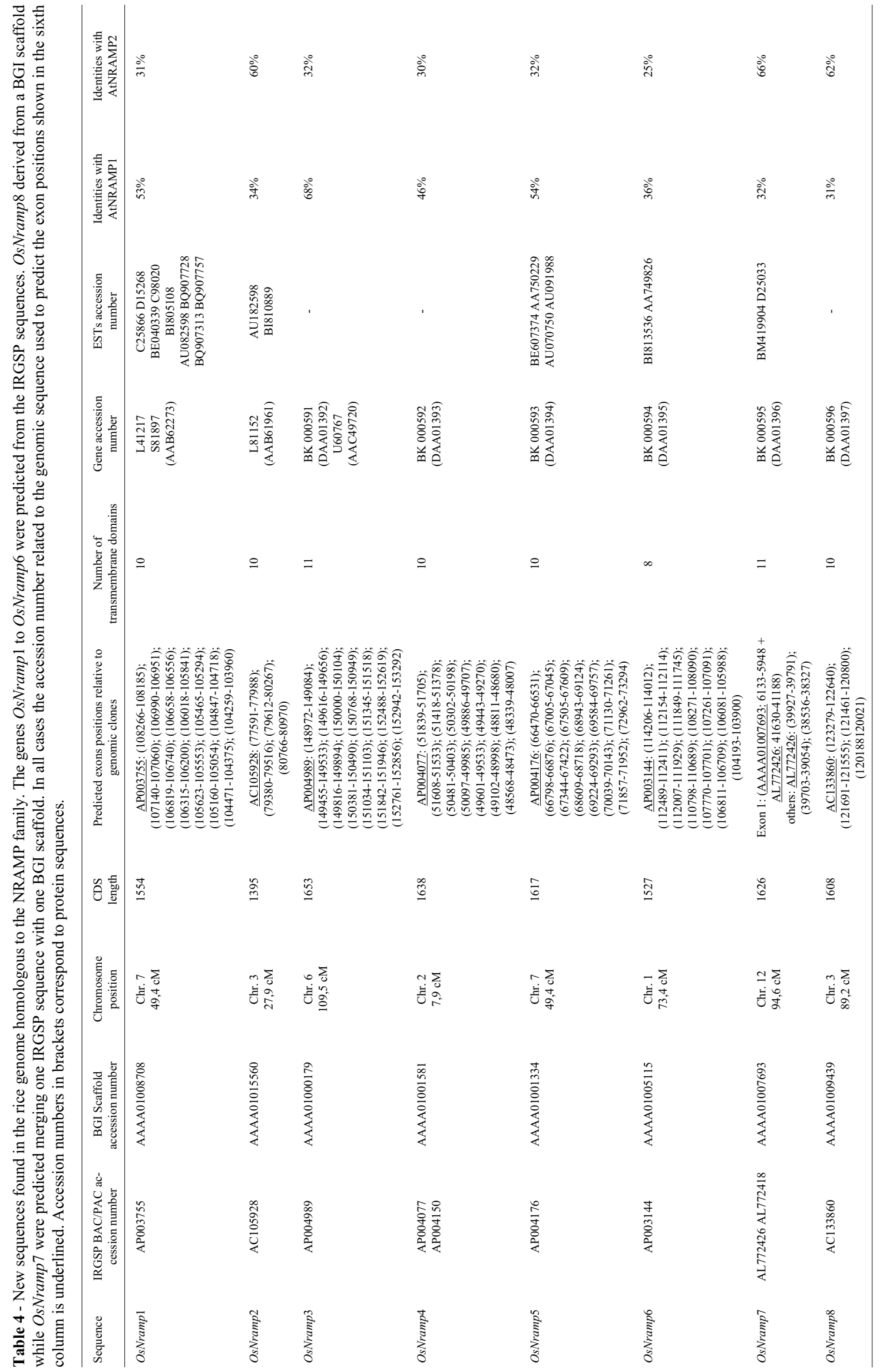




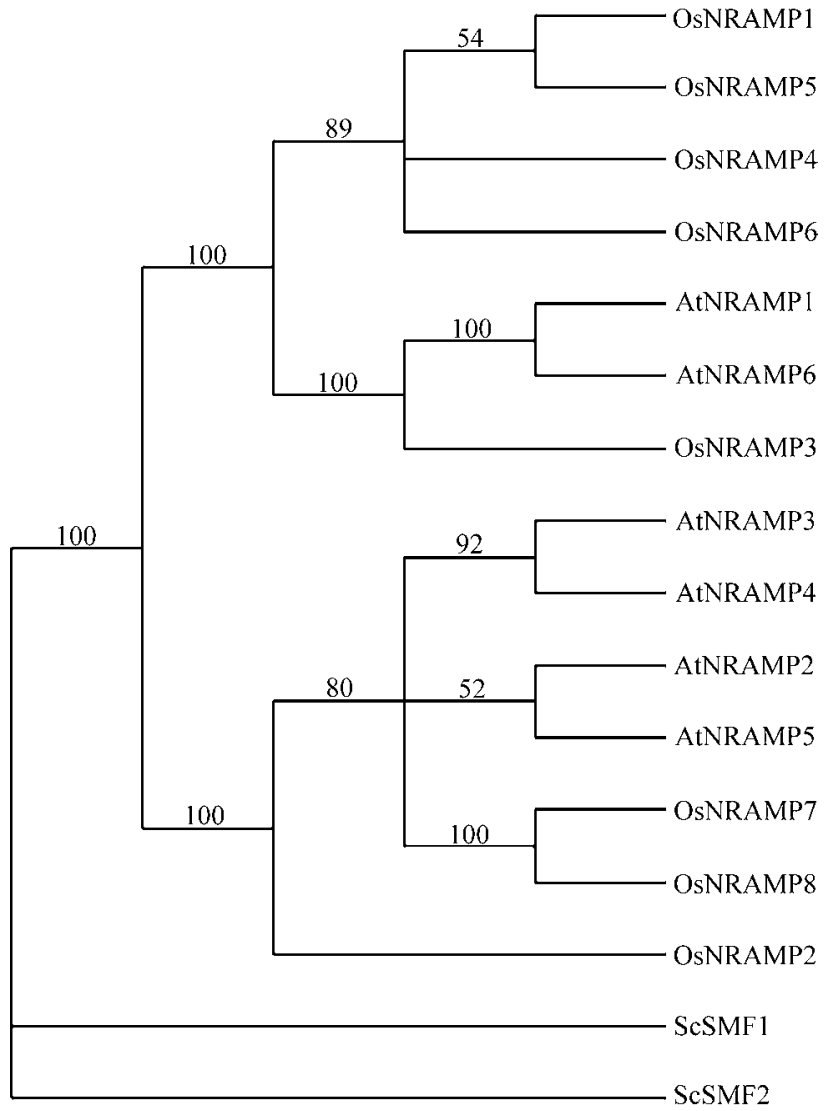

Figure 6 - Phylogenetic analysis of the NRAMP protein family. The new OsNRAMP proteins were analyzed together with the NRAMP proteins from Arabidopsis and the $S$. cerevisiae SMF1 and SMF2 polypeptides. Accession numbers of nucleotide sequences and corresponding predicted OsNRAMP proteins are shown in Table 4. Accession numbers to the other proteins are: AtNRAMP1: AAF36535; AtNRAMP2: AAD41078; AtNRAMP3: NP_179896; AtNRAMP4: AAF13279; AtNRAMP5: NP 193614; AtNRAMP6: NP 173048; ScSMF1: AAB48984; ScSMF2: B45 $\overline{5} 154$.

sequences, but it is not known if they are functional (Petit $e t$ al., 2001b).

Post-transcriptional regulation of plant ferritin genes has also been shown, with accumulation of ferritin mRNA but not protein in the maize $y_{s} 1$ mutant (with low iron content in leaves) treated with iron (Fobis-Loisy et al., 1996). Expression of the soybean ferritin gene in rice seeds resulted in up to a threefold increase in iron content (Goto et al., 1999).

The search for sequences homologous to the maize ferritin (ZmFER1) yielded several related sequences in rice. These include 20 ESTs, 2 sequenced cDNAs, 2 genomic sequences from the IRGSP and 4 BGI scaffolds (Table 5). A comparative analysis of ESTs showed two distinct patterns of expressed genes, which differ in the 5 'region by point variations and two small deletions. In order to better analyze these differences, we sequenced two cDNAs from the japonica variety, corresponding to ESTs D22266 and AU064475, representing the two observed patterns. The full length cDNA information confirmed the

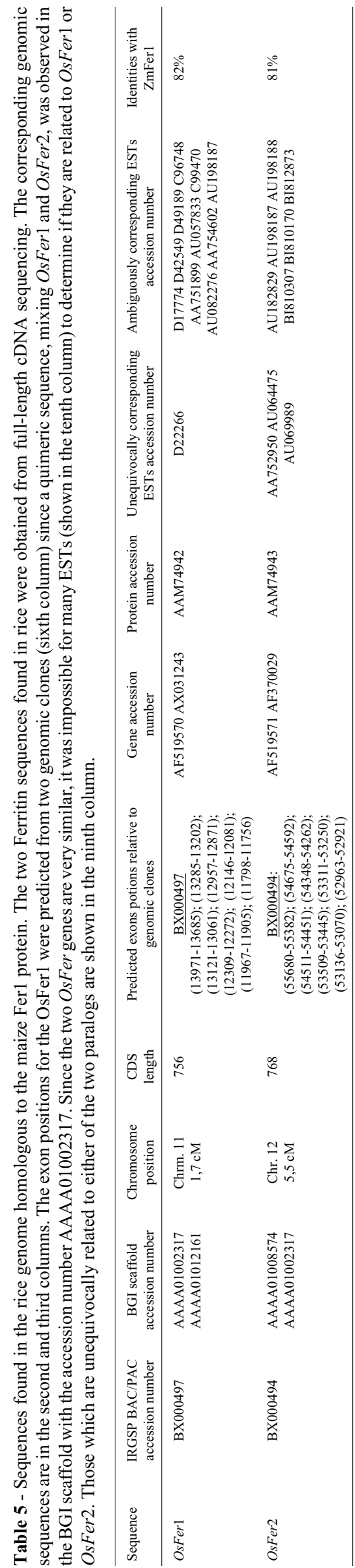


two distinct gene patterns, which were named OsFER1 and OSFER2. The new genes are very similar, with $96 \%$ identity. In addition to 15 SNPs along the complete cDNA, OsFER1 has two deletions of $12 \mathrm{bp}$ not seen in OSFER2. One of the deletions is located in the 5'untranslated region and the other immediately downstream from the start codon. Due to the high similarity between the two sequences, very few ESTs found in the data banks could be unequivocally related to one of the gene types (Table 5, column 9). OsFER2 is related to the BX000494 genomic sequence of the IRGSP and to two BGI scaffolds. The 5 'region of OsFER1, containing the two deletions characteristic of this sequence, is found in the final part of the AAAA01012161 scaffold, whereas the rest of the gene is found in scaffold AAAA01002317. Eight exons were found in the genomic sequences OSFER1 and OsFER2, which is the number found in all plant ferritins described so far.

The CDS predicted from scaffold AAAA01002317 has similarities with portions of both genes that have been identified based on expressed sequences. There is identity with OsFER2 in the 19513-19409 region of the referred scaffold, as seen from the SNPs and insertions found in the OSFER 2 sequence relative to OSFER 1 . From the 19410 position of scaffold AAAA 01002317 onwards, all SNPs correspond to those observed in OSFER1. This is possibly due to a chimera in the assembly of the scaffold, which joined sequences derived from two very similar loci, one encoding $O S F E R 2$, followed by another coding for $O S F E R 1$. Errors in the computational assembly of sequences involving similar regions in the genome have been pointed out as the main disadvantage of the total shotgun sequencing methodology (Waterston et al., 2002), employed by BGI.

The OsFER 1 and OsFER 2 proteins have 82 and $81 \%$ identity with the reference polypeptide ZmFER1, respectively, and share $96 \%$ identity. The protein alignment of OsFER 1 and OsFER2 to six plant ferritins and to the structures of equine Heavy Chain Ferritin and human $\mathrm{H}$ ferritin (tridimensional structure already defined) is shown in Figure 7. The rice ferritins have more than $30 \%$ identity in relation to the mammalian ferritins and between 58 and $82 \%$ with other plant ferritins. A transit peptide and an extension peptide are only seen in plant ferritins. The transit peptide is involved in the subcellular localization of ferritin to the plastid in maize, soybean and pea (Briat and Lobréaux, 1998). The limits of the transit peptide indicated in Figure 7 are based on the extension observed in Ferritin 1 of maize (Fobis-Loisy et al., 1995). The cleavage of the transit peptide generates a mature protein that has the so called extension peptide, which represents an extra portion not seen in the mammalian ferritins (Briat and Lobréaux, 1998). The latter are composed of a bundle of 5 alpha helices forming a subunit. Twenty-four of these subunits compose a spherelike holoprotein, which has channels or specific pores formed by the junction of 3 to 4 subunits (Harrison et al.,
1998). The 3 -fold channel (3FC) is formed by the junction of 3 subunits and is functionally related to iron incorporation. The amino acids related to the structure of this channel are hydrophilic and are identical in almost all ferritins aligned in Figure 7. The 4-fold channel (4FC) is formed by the junction of 4 different subunits. In mammalian ferritins, this channel is hydrophobic in nature and is presumably related to the release of iron from the holoprotein. The amino acids related to the $4 \mathrm{FC}$ and the remaining residues that compose the $\mathrm{E}$ helix are not much conserved between ferritins of mammals and plants, although in the latter the iron release mechanism is also related to the E-helix through its proteolytic cleavage (Masuda et al., 2001). Amino acid residues related to the catalytic iron oxidation (FO) in $\mathrm{H}$ ferritin are conserved in plant ferritins, indicating a possible catalytic role for these residues also in plant ferritins (Briat and Lobréaux, 1998; Harrison et al., 1998).

The same ClustalW alignment in Figure 7 was used as starting point for the phylogenetic analysis shown in Figure 8. The depicted tree was obtained after 542 steps of a branch and bound search. The resulting consistency index was 0.9059 . The dendrogram separates the mammalian ferritins from their plant homologs. Within the plant ferritins, the four Arabidopsis sequences are placed in a separate clade from the grass ferritins. In a similar fashion, within the grass ferritins, there is a division separating the rice sequences from those of corn.

Analysis of the promoter regions of the newly described rice ferritin genes revealed the presence of the IDRS (Iron-Dependent Regulatory Sequence) in both of them. The sequence (CACGAGCCCGCCAC) is the same in OsFerl and $O s F e r 2$, and has one base difference in relation to both the AtFerl IDRS (C instead of G in the seventh position) and the ZmFerl IRDS (G instead of $\mathrm{T}$ in the tenth position) (Petit et al., 2001a). Interestingly, these are the only two bases in which there are differences between the two previously reported IRDS, and the one found in rice shares one of the bases with maize and the other one with Arabidopsis, establishing a trend to a consensus sequence CACGAG( $\mathrm{C}$ or $\mathrm{G}) \mathrm{CC}(\mathrm{G}$ or T)CCAC. Experimental data is needed to characterize the functionality of this element and its responsiveness to iron levels in regulating expression of the ferritin genes in rice.

\section{Multiplicity of transporters and members in the gene families}

Mineral homeostasis in plants is just starting to be analyzed at the molecular level. A number of studies have shown that there are several types of transporters and homologs of each of them in Arabidopsis and other plants (Williams et al., 2000; Maser et al., 2001). The multiple DNA sequences related to iron homeostasis in rice found in this study is in agreement with the diversity observed in other species. What are the biological implications of different protein families involved in the transport of the same 


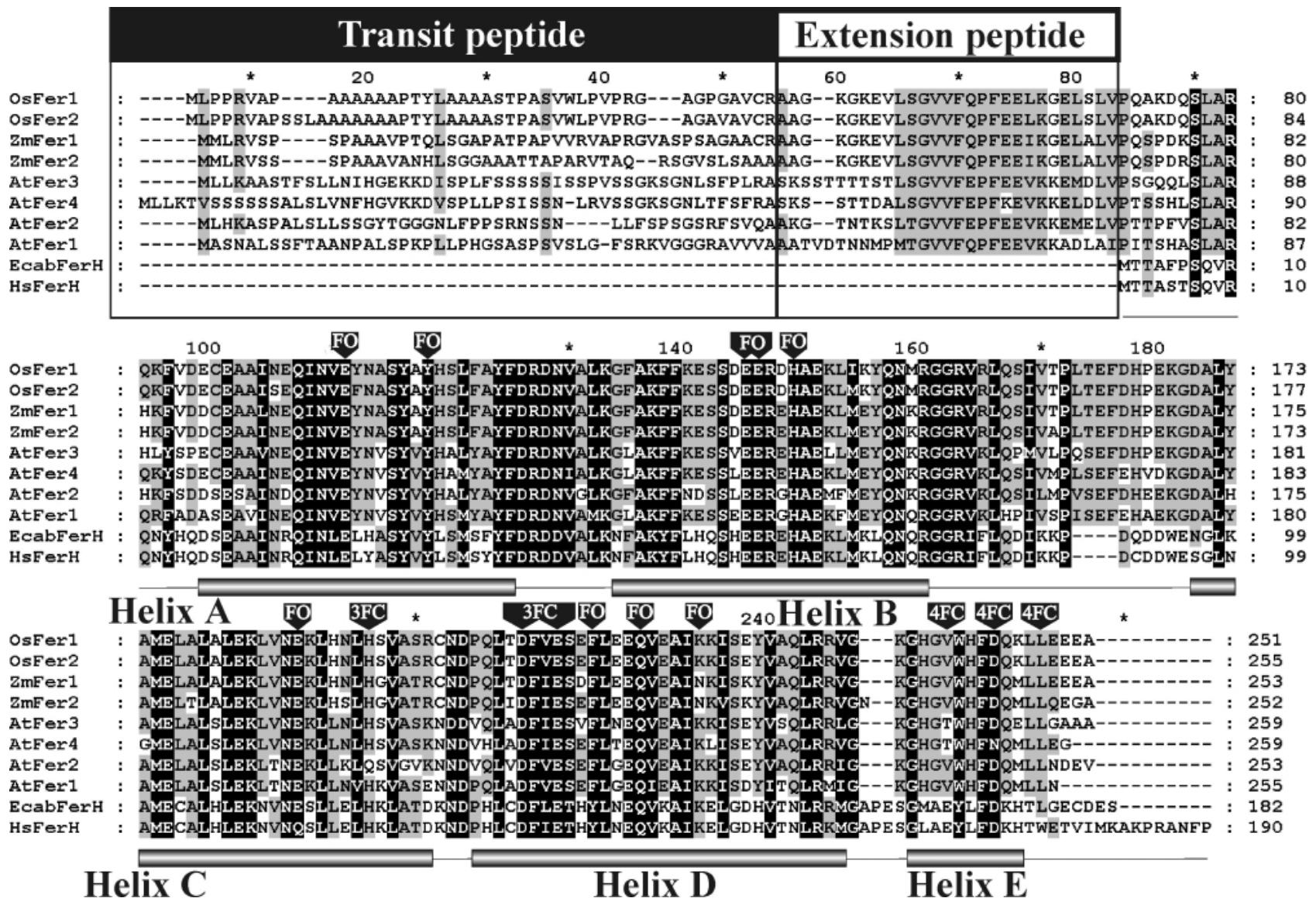

Figure 7 - ClustalW multiple alignment of the Ferritin proteins. The figure shows the structure of plant Ferritins of Oryza sativa (OsFer1: AAM74942; OsFer2: AAM74943), Zea mays (ZmFer1: CAA58146; ZmFer2: CAA58147), Arabidopsis thaliana (AtFer1: CAA63932; AtFer2: CAC85498; AtFer3: CAC85399; AtFer4: CAC85400) and compares them with the mammalian homologues of Homo sapiens heavy chain (HsFerH: CAA25086) and Equus caballus heavy chain (EcabFerH: AAM51631). A transit peptide and an extension peptide are delimited in the corresponding boxes. Alpha helixes are positioned below the alignment block according to the extensions from the HsFerH. Some functional aminoacids are pointed above the alignment block in the positions that they occur in the HsFerH. In this protein they are involved in the Ferroxidase catalytic center (FO), constitution of the 3-fold channel $(3 \mathrm{FC})$ and the 4-fold channel (4FC).

mineral and the occurrence of various paralogs within a family of transporters?

Initially, distinct iron transporter families were explained on the basis of the different $\mathrm{Fe}$ uptake strategies (Guerinot and Yi, 1994). In strategy I, found in dicots and non-graminaceous monocots, after the solubilization and reduction steps, $\mathrm{Fe}^{2+}$ would be taken up by a specific transporter, later on characterized as IRT1 (Eide et al., 1996). In contrast, grasses would take up $\mathrm{Fe}^{3+}$ in the form of complexes with phytosiderophores by another class of transporters, later defined as Yellow Stripe1 (Curie et al., 2001). With the complete genomic sequence of Arabidopsis, it was found that eight homologs of the grass YS transporter were present in this dicot. In the present study, it was also observed that there are IRT homologs in the rice genome. Therefore, the notion that a specific family of transporters is related to a certain physiological strategy for iron uptake, although real, seems not to be exclusive and must be revised.

The presence of YS family members in Arabidopsis, a species that does not produce phytosiderophores, has been explained on the basis of nicotianamine (NA) transport (Curie et al., 2001). This compound is ubiquitous in plant tissues and is a structural analog of the derivatives of mugineic acid (MA), the phytosiderophores. It has been proposed that NA plays a fundamental role in iron homeostasis, as a transport form for minerals in the phloem (Stephan and Scholtz, 1993). The corn YS1 transporter is capable of transporting $\mathrm{Fe}^{2+}$-nicotianamine complexes based on yeast functional complementation assays (Walker et al., 2001). Immunodetection studies showed an increase of NA-chelated iron in tomato tissues exposed to excess concentration of the metal (Pich et al., 2001). Therefore, the YS transporters in dicots could play a role in the transport of NA-complexed minerals, not being involved in uptake, whereas the ZIP family of transporters would be responsible for $\mathrm{Fe}^{2+}$ uptake in roots, with a possible additional function in micronutrient distribution throughout the plant.

What about the rationale for the presence of IRT orthologs in grasses such as rice? The mechanism based on 
the release of phytosiderophores for $\mathrm{Fe}^{+3}$ chelation and uptake under mineral deficiency represents an evolutionary advantage relative to the mechanism of strategy I, since the latter requires an energy dependent reduction step prior to the uptake (Grusak et al., 1999). Presumably, grasses acquired the strategy II components in addition to those of strategy I, which progressively assumed a more secondary role in mineral uptake and transport. The larger number of YS orthologs in rice (18) compared to ZIP orthologs (13) supports the greater importance of YS in mineral uptake and transport. The opposite scenario is seen in Arabidopsis, in which there are fifteen ZIP members and only eight YS orthologs, in agreement with a predominant role for the ZIP family in this species. The recently cloned OSIRT1 in rice, expressed in roots under mineral deficiency and capable of rescuing iron deficient yeast mutants, has been proposed as an actively engaged iron uptake system, especially under limiting conditions (Bughio et al. 2002). The functional characterization of OsIrt1 is evidence that rice also possesses an $\mathrm{Fe}^{2+}$ uptake system that could be involved in ferrous ion absorption under waterlogged conditions, in which this form of iron is prevalent. This result suggests that grasses may not be restricted to a single strategy for iron uptake, but can adjust the system according to environmental requirements (Bughio et al., 2002).

The NRAMP family members coexist with YS and ZIP families in Arabidopsis and, as seen in this study, in rice. Except for a suggested role for AtNRAMP3 (Thomine et al., 2000), the NRAMP proteins do not seem to be involved in iron uptake from the soil, but probably function in the subcellular transport of the mineral. Transgenic Arabidopsis lines overexpressing NRAMP1 showed higher resistance to toxic concentrations of iron than wild type plants, proportionately to the transgene expression. Resistance could be caused by iron relocation, promoted by the NRAMP1 protein, from the cytosol to the vacuole or the plastids (Curie et al., 2000).

Another reason for the presence of three different families of iron transporters in plants could be differential affinity for iron in different oxidation states. The NRAMP family is known as a transporter for divalent cations (Williams et al., 2000). Similarly, IRT1 has affinity for $\mathrm{Fe}^{2+}$ (Eide et al., 1996), whereas only the YS transporter would be capable of ferrying the cation in its ferric form (Curie et al., 2001). The availability of various transporters may fulfill the needs for iron transport in different redox environments or under fluctuating redox conditions.

Different iron carrier molecules may also explain the diversity of iron transporter families in plants. The YS1 protein, for example, transports $\mathrm{Fe}^{3+}$ phytosiderophores, and possibly $\mathrm{Fe}^{+2}$ NA (Walker et al., 2001), structurally similar to phytosiderophores. Another important mineral carrier in plants is citrate, mainly associated with iron transport in the xylem (Grusak et al., 1999). Since members of the YS family were unable to transport citrate-complexed

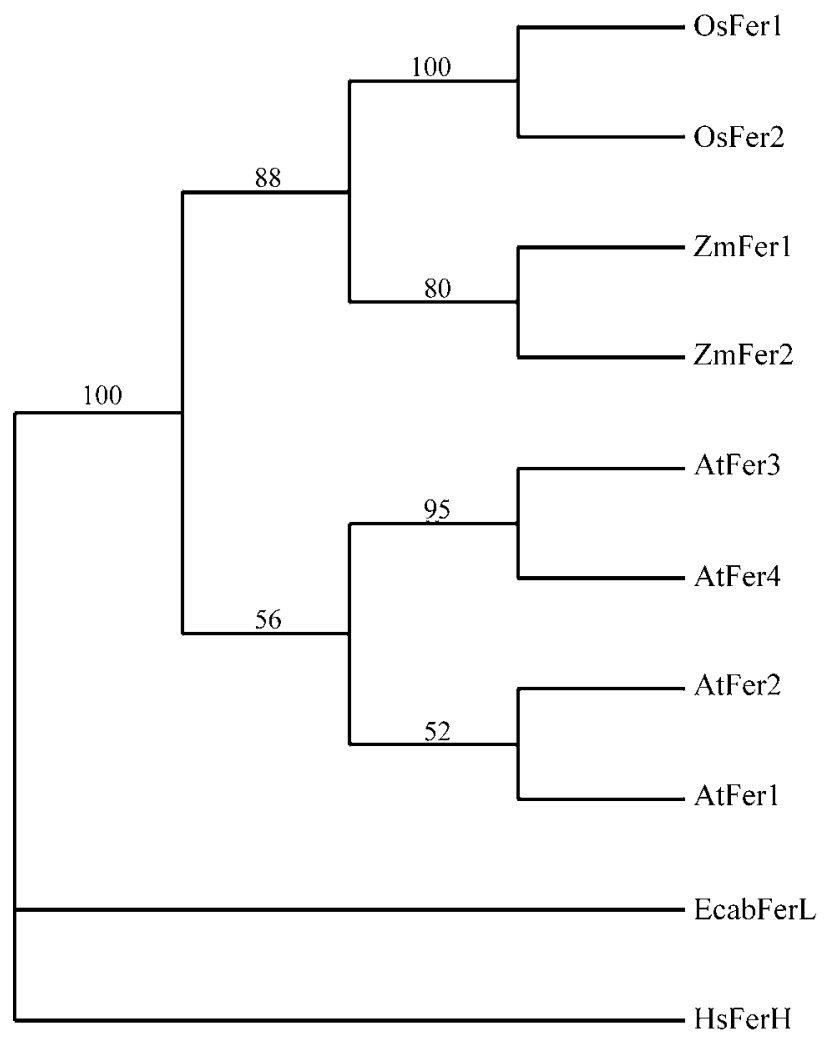

Figure 8 - Phylogenetic relationships of Oryza sativa Ferritins to plant and mammalian Ferritins. The proteins are from Oryza sativa (OsFer1: AAM74942; OsFer2: AAM74943), Zea mays (ZmFer1: CAA58146; ZmFer2: CAA58147), Arabidopsis thaliana (AtFer1: CAA63932; AtFer2: CAC85498; AtFer3: CAC85399; AtFer4: CAC85400) and the mammalian homologues Homo sapiens heavy chain (HsFerH: CAA25086) and Equus caballus heavy chain (EcabFerH: AAM51631).

minerals (Curie et al., 2001), it may be possible that NRAMP or ZIP members could transport such complexes.

The variety of paralogs found within each family of transporters could be explained by at least three reasons: 1) paralogs with different affinities for different minerals or distinct affinities for the same mineral; 2) different paralogs operating in the various organs and tissues; 3 ) different paralogs for each subcellular compartment, such as vacuoles or plastids. Yeast complementation assays showed that plant mineral transporters are able to transport several minerals, often displaying preferences for the transport of one or more of these.

The IRT proteins are mostly involved in iron transport. Although with lower affinity, Arabidopsis IRT1 was also capable of transporting $\mathrm{Zn}^{2+}, \mathrm{Mn}^{2+}$ and $\mathrm{Cd}^{2+}$, as shown by radioisotope and functional complementation assays (Eide et al., 1996; Korshunova et al., 1999; Rogers et al., 2000). Similarly, Arabidopsis IRT2 seems to be specific for iron and $\mathrm{Zn}^{2+}$ transport, not showing affinity for other cations (Vert et al., 2001). Tomato IRT1 and 2 may also be capable of transporting zinc, copper and manganese (Eckhardt et al., 2001). On the other hand, ZIP1 through 4 show specificity for zinc transport, but not for iron. Zinc 
uptake by ZIP2-complemented yeast was strongly inhibited by $\mathrm{Cd}^{2+}$ and $\mathrm{Cu}^{2+}$ (Grotz et al., 1998). It is quite possible that similar features are found in the rice ZIP family members. The plant NRAMP family members have been characterized for the transport of iron and cadmium. Expression of AtNramp 1, 3 and 4 resulted in increased cadmium sensitivity and cadmium content in yeast, and the same genes can also complement a yeast mutant defective in manganese uptake (Thomine et al., 2000). Moreover, mammalian and yeast orthologs have a wide spectrum of mineral transport (Williams et al., 2000). This may lead to the hypothesis that the eight rice NRAMP members could be partly explained by diverse affinities of the individual members for different minerals. The important role of NA in mineral homeostasis may be one of the reasons for the large number of YS family members in the rice genome. NA is well known for its capacity to chelate a variety of minerals (Benes et al., 1983), being involved in the transport of copper in the phloem and possibly performing the same function for zinc, iron and manganese (Stephan and Scholtz et al., 1993). In this regard, it is reasonable to suggest that different YS paralogs may coordinate the transport of various NA-metal complexes. The release of phytosiderophores is not only stimulated by iron deficiency, but by zinc as well (Tolay et al., 2001), indicating that this mineral may also be transported in complexed form with phytosiderophores, perhaps by a YS paralog.

Different paralogs may also have different affinities for the same cation. In yeast, the ZIP orthologs constitute two systems with different affinities for zinc. ZRT1 has high affinity for the metal, being active when zinc is limiting in the cell, whereas ZRT2 shows low affinity, transporting the mineral when it is abundant in the environment (Guerinot, 2000). Yeast complementation assays indicated that Arabidopsis ZIP1, 2, and 3 have different zinc uptake activities, with $\mathrm{Km}$ values ranging from $2 \mu \mathrm{M}$ in ZIP2 to 14 $\mu \mathrm{M}$ zinc in ZIP3 (Grotz et al., 1998). Such different affinities for the mineral may also occur in the rice ZIP, NRAMP, and YS families, constituting a possible explanation for the diversity of paralogs observed in this species.

The mineral flow throughout the plant requires the transport across barriers that delineate tissue compartments of long distance transport, such as xylem and phloem, and cell compartments in other plant tissues. Therefore, different transporters could be associated with specific tissues or cell types. Northern blot experiments have provided strong evidence that paralog diversity is related to their expression in different organs and tissues. In rice, NRAMP1 is expressed in roots, whereas $N R A M P 2$ is expressed in leaves and NRAMP3 in both organs (Belouchi et al., 1997). In Arabidopsis, organ-specific expression is also observed. NRAMP1 and 2 are preferentially expressed in roots, whereas $N R A M P 3$ and 4 are equally expressed in roots and shoots. Moreover, although NRAMP3 expression occurs in shoots under iron availability, expression under iron defi- ciency is only induced in roots for this paralog (Thomine et al., 2000). Arabidopsis IRT1 and IRT2 are exclusively expressed in roots (Eide et al., 1996; Vert et al., 2001), the same happening with ZIP1 and ZIP3 of the same family. ZIP4 is expressed under iron deficiency in shoots and roots (Grotz et al., 1998). The EST patterns found in this study for the rice YS family are in good agreement with these data on organ-specific expression. The ESTs that make up the OsYS4 pattern were obtained from cDNA libraries derived from flowering panicules, whereas ESTs related to the OsYS6 paralog originated in cDNA libraries built from roots of seedlings.

Besides the need for organ or tissue specific transporters, it is possible that different isoforms of the iron reducing enzymes are also required. Under limited iron availability, AtFROHC and PsFRO1 were shown to be expressed in leaves, evidence that FRO members may act in a reducing step before iron moving through the xylem is absorbed by leaves.

Some paralogs observed in the various transporter families may also be related to the subcellular transport of iron and other minerals. The varying patterns of expression of different Nramp genes in plants are consistent with possible localization in distinct membranes in the plant cell, such as the plasma membrane, the tonoplast and the chloroplast envelope. AtNRAMP1 could be involved in the sequestration of free cytosolic iron into the plastids or the vacuole (Curie et al., 2000). The subcellular localization of each one of the isoforms will help to clarify this point. A role in internal iron transport to a subcellular location has also been proposed for IRT2, since its constitutive expression could not suppress the defect in iron uptake from the Arabidopsis Irt1 T-DNA mutant (Vert et al., 2002). On the basis of a predicted transit peptide, it is also believed that Arabidopsis ZIP4 may function as a zinc transporter to the plastid. The subcellular localization signal predictions conducted in the present study pointed toward some evidence of plastid localization of OsYS12 and OsFer2 encoded in chromosome 12, mitochondrial localization of OsZIP1 and OsNRAMP2, as well as involvement in secretory pathways for OsNRAMP5. These and other proteins may be responsible for coordinating influx and possible efflux of minerals in subcellular compartments.

\section{Perspectives}

The novel putative components of iron homeostasis in rice reported in this study open new perspectives in the study of iron and other mineral nutrients uptake and distribution in this important crop species. More refined studies, involving complementation analysis in yeast strains deficient in iron acquisition, gene expression analysis, immunodetection and protein level analyses, may define the individual role that each of these new transporters plays in mineral homeostasis, as well as provide the basis for efficient biotechnological manipulation of iron content in rice 
plants. We are currently investigating the expression patterns of several of these new genes in rice plants exposed to varying levels of iron. We are especially interested in comparing expression between rice varieties considered resistant and susceptible to iron toxicity under waterlogged conditions.

\section{Acknowledgments}

This work was supported by FAPERGS (grant 02/0503.4 to J.P.F.), CAPES (graduate scholarship to J.G.) and $\mathrm{CNPq}$ (fellowships to A.G.F.-N. and J.P.F. and undergraduate scholarship to R.J.S.). We thank Dr. Tatiana Chies (Department of Botany, UFRGS) for help with the phylogenetic analysis, M.Sc. Deise Porto Potrich (Center of Biotechnology, UFRGS) for help with automatic DNA sequencing and the MAFF DNA Bank (National Institute of Agrobiological Resources, Japan) for kindly providing the EST clones sequenced in this work.

\section{References}

Altschul SF, Madden TL, Schaffer AA, Zhang J, Zhang Z, Miller W and Lipman DJ (1997) Gapped BLAST and PSI-BLAST: a new generation of protein database search programs. Nucleic Acids Res 25:3389-3402.

Bar-Ness E, Hadar Y, Chen Y, Romheld V and Marschner H (1992) Short-term effects of rhizosphere microorganisms on Fe uptake from microbial siderophores by maize and oat. Plant Physiol 100:451-456.

Belouchi A, Kwan T and Gros P (1997) Cloning and characterization of the OsNramp family from Oryza sativa, a new family of membrane proteins possibly implicated in the transport of metal ions. Plant Mol Biol 33:1085-1092.

Benes I, Schreiber K, Ripperger H and Kircheiss A (1983) Metal complex formation by nicotianamine, a possible phytosiderophore. Experimentia 39:261-262.

Briat JF, Fobis-Loisy I, Grignon N, Lobréaux S, Pascal N, Savino G, Thoiron S, von Wirén N and van Wuytswinkel O (1995) Cellular and molecular aspects of iron metabolism in plants. Biol Cell 84:69-81.

Briat JF and Lobréaux S (1997) Iron transport and storage in plants. Trends Plant Sci 2:187-193.

Briat JF and Lobréaux S (1998) Iron storage and ferritin in plants. In: Sigel A and Sigel H (eds) Metal Ions in Biological Systems: Iron Transport and Storage in Microorganisms, Plants and Animals, Marcel Dekker Inc, New York, pp 563-584.

Bughio N, Yamaguchi H, Nishizawa NK, Nakanishi H and Mori S (2002) Cloning an iron-regulated metal transporter from rice. J Exp Bot 53:1677-1682.

Burge C and Karlin S (1997) Prediction of complete gene structures in human genomic DNA. J Mol Biol 268:78-94.

Burr B (2002) Mapping and Sequencing the Rice Genome. Plant Cell 14:521-523.

Cellier M, Privé G, Belouchi A, Kwan T, Rodriques V, Chia W and Gros P (1995) Nramp defines a family of membrane proteins. Proc Natl Acad Sci USA 92:10089-10093.

Curie C, Alonso JM, Le Jean M, Ecker JR and Briat JF (2000) Involvement of NRAMP1 from Arabidopsis thaliana in iron transport. Biochem J 347:749-755.
Curie C, Panaviene Z, Loulergue C, Dellaporta SL, Briat JF and Walker EL (2001) Maize yellow stripel encodes a membrane protein directly involved in Fe(III) uptake. Nature 409:346-349.

Chaney RL, Brown, JC and Tiffin LO (1972) Obligatory reduction of ferric chelates in iron uptake by soybean. Plant Physiol 50:208-213.

Connolly EL, Fett JP and Guerinot ML (2002) Expression of the IRT1 metal transporter is controlled by metals at the levels of transcript and protein accumulation. Plant Cell 14:13471357.

Dancis A, Roman DG, Anderson GJ, Hinnebusch AG and Klausner RD (1992) Ferric reductase of Saccharomyces cerevisiae: molecular characterization, role in iron uptake, and transcriptional control by iron. Proc Natl Acad Sci USA 89:3869-3873.

Delseny M, Salses J, Cooke R, Sallaud C, Regad F, Lagoda P, Guiderdoni E, Ventelon $\mathrm{M}$, Brugidou $\mathrm{C}$ and Ghesquière A (2001) Rice genomics: present and future. Plant Physiol Biochem 39:323-334.

Eckardt NA (2000) Sequencing the rice genome. Plant Cell 12:2011-2017.

Eckhardt U, Marques AM and Buckhout TJ (2001) Two ironregulated cation transporters from tomato complement metal uptake-deficient yeast mutants. Plant Mol Biol 45:437-448.

Eide D, Broderius M, Fett JP and Guerinot ML (1996) A novel iron-regulated metal transporter from plants identified by functional expression in yeast. Proc Natl Acad Sci USA 93:5624-5628.

Fobis-Loisy I, Loridon K, Lobreaux S, Lebrun M and Briat JF (1995) Structure and differential expression of two maize ferritin genes in response to iron and abscisic acid. Eur $\mathrm{J}$ Biochem 231:609-619.

Fobis-Loisy I, Aussel L and Briat J-F (1996) Post-transcriptional regulation of plant ferritin accumulation in response to iron as observed in the maize mutant $y s 1$. FEBS Letters 397:149-154.

Fox TC, Shaff JE, Grusak MA, Norvell WA, Chen Y, Chaney RL and Kochian LV (1996) Direct measurement of ${ }^{59} \mathrm{Fe}$-labeled $\mathrm{Fe}^{2+}$ influx in roots of pea using a chelator buffer system to control free $\mathrm{Fe}^{2+}$ in solution. Plant Physiol 111:93-100.

Goff SA, Ricke D, Lan T-H, Presting G, Wang R, Dunn M, Glazebrook J, Sessions A, Oeller P, Varma H, Hadley D, Hutchison D, Martin C, Katagiri F, Lange BM, Moughamer T, Xia Y, Budworth P, Zhong J, Miguel T, Paszkowski U, Zhang S, Colbert M, Sun W, Chen L, Cooper B, Park S, Wood TC, Mao L, Quail P, Wing R, Dean R, Yu Y, Zharkikh A, Shen R, Sahasrabudhe S, Thomas A, Cannings R, Gutin A, Pruss D, Reid J, Tavtigian S, Mitchell J, Eldredge G, Scholl T, Miller RM, Bhatnagar S, Adey N, Rubano T, Tusneem N, Robinson R, Feldhaus J, Macalma T, Oliphant A and Briggs S (2002) A draft sequence of the rice genome (Oryza sativa L. ssp. japonica). Science 296:92-100.

Groom QJ, Torres MA, Fordham-Skelton AP, Hammond-Kosack KE, Robinson NJ and Jones JD (1996) rbohA, a rice homologue of the mammalian gp91phox respiratory burst oxidase gene. Plant J 10:515-522.

Grotz N, Fox T, Connolly E, Park W, Guerinot ML and Eide D (1998) Identification of a family of zinc transporter genes 
from Arabidopsis that respond to zinc deficiency. Proc Natl Acad Sci USA 95:7220-7224.

Grotz N and Guerinot ML (2002) Limiting nutrients: an old problem with new solutions? Curr Opin Plant Biol 5:158-163.

Grusak MA, Pearson JN and Marentes E (1999) The physiology of micronutrient homeostasis in field crops. Field Crops Res 60:41-56

Grusak, MA (2002) Enhancing mineral content in plant food products. J Am Coll Nutr 21:178S-183S.

Guerinot ML and Yi Y (1994) Iron: nutritious, noxious, and not readily available. Plant Physiol 104:815-820.

Guerinot ML (2000) The ZIP family of metal transporters. Biochim Biophys Acta 1465(1-2):190-198.

Guerinot ML (2001) Improving rice yields-ironing out the details. Nat Biotechnol 19:417-418.

Harrison PM, Hempstead PD, Artymiuk PJ and Andrews SC (1998) Structure-function relationships in the ferritins. In: Sigel A and Sigel H (eds) Metal Ions in Biological Systems: Iron Transport and Storage in Microorganisms, Plants and Animals, Marcel Dekker Inc, New York, pp 435-477.

Hauser M, Donhardt AM, Barnes D, Naider F and Becker JM (2000) Enkephalins are transported by a novel eukaryotic peptide uptake system. J Biol Chem 275:3037-3041.

Hauser M, Narita V, Donhardt AM, Naider F and Becker JM (2001) Multiplicity and regulation of genes encoding peptide transporters in Saccharomyces cerevisiae. Mol Membr Biol 18:105-112.

Hirokawa T, Boon-Chieng S and Mitaku S (1998) SOSUI: Classification and secondary structure prediction system for membrane proteins. Bioinformatics 14:378.

Koh S, Wiles AM, Sharp JS, Naider FR, Becker JM and Stacey G (2002) An oligopeptide transporter gene family in Arabidopsis. Plant Physiol 128:21-29.

Korshunova YO, Eide D, Clark WG, Guerinot ML and Pakrasi HB (1999) The IRT1 protein from Arabidopsis thaliana is a metal transporter with a broad substrate range. Plant Mol Biol 40:37-44

Lubkowitz MA, Hauser L, Breslav M, Naider F and Becker JM (1997) An oligopeptide transport gene from Candida albicans. Microbiology 143:387-396.

Marchler-Bauer A, Panchenko AR, Shoemaker BA, Thiessen PA, Geer LY and Bryant SH (2002) CDD: a database of conserved domain alignments with links to domain threedimensional structure. Nucleic Acids Res 30:281-283.

Marschner H and Römheld V (1994) Strategies of plants for acquisition of iron. Plant Soil 165:261-274.

Mäser P, Thomine S, Schroeder JI, Ward JM, Hirschi K, Sze H, Talke IN, Amtmann A, Maathuis FJ, Sanders D, Harper JF, Tchieu J, Gribskov M, Persans MW, Salt DE, Kim SA and Guerinot ML (2001) Phylogenetic relationships within cation transporter families of Arabidopsis. Plant Physiol 126:1646-1667.

Masuda T, Goto F and Yoshihara T (2001) A novel plant ferritin subunit from soybean that is related to a mechanism in iron release. J Biol Chem 276:19575-19579.

Moog PR and Brüggermann W (1994) Iron reductase systems on the plant plasma membrane- a review. Plant Soil 165:241260

Morgenstern B (1999) DIALIGN 2: improvement of the segment-to-segment approach to multiple sequence alignment. Bioinformatics 15:211-218.
Moreau S, Thomson RM, Kaiser BN, Trevaskis B, Guerinot ML, Udvardi MK, Puppo A and Day DA (2002) GmZIP1 encodes a symbiosis-specific zinc transporter in soybean. $\mathrm{J}$ Biol Chem 277:4738-4746.

Murray-Kolb LE, Takaiwa F, Goto F, Yoshihara T, Theil EC and Beard JL (2002) Transgenic rice is a source of iron for iron-depleted rats. J Nutr 132:957-960.

Orengo CA, Jones DT and Thornton JM (1994) Protein superfamilies and domain superfolds. Nature 372:631-634.

Petit J-M, van Wuytswinkel O, Briat J-F and Lobréaux S (2001a) Characterization of an iron-dependent regulatory sequence involved in the transcriptional control of AtFerl and ZmFerl plant ferritin genes by iron. J Biol Chem 276:55845590.

Petit J-M, Briat J-F and Lobreaux S (2001b) Structure and differential expression of the four members of the Arabidopsis thaliana ferritin gene family. Biochem J 359:575-582.

Pich A, Manteuffel R, Hillmer S, Scholz G and Schmidt W (2001) Fe homeostasis in plant cells: Does nicotianamine play multiple roles in the regulation of cytoplasmic Fe concentration? Planta 213:967-976.

Ragland M, Briat JF, Gagnon J, Laulhere JP, Massenet $\mathrm{O}$ and Theil EC (1990) Evidence for conservation of ferritin sequences among plants and animals and for a transit peptide in soybean. J Biol Chem 265:18339-18344.

Robinson NJ, Sadjuga and Groom QJ (1997) The froh gene family from Arabidopsis thaliana: Putative iron-chelate reductases. Plant and Soil 196:245-248.

Robinson NJ, Procter CM, Connolly EL and Guerinot ML (1999) A ferric-chelate reductase for iron uptake from soils. Nature 397:694-697.

Rogers EE, Eide DJ and Guerinot ML (2000) Altered selectivity in an Arabidopsis metal transporter. Proc Natl Acad Sci USA 97:12356-12360.

Römheld V and Marschner H (1983) Mechanism of iron uptake by peanut plants. I- Fe(III) reduction, chelate splitting, and release of phenolics. Plant Physiol 71:949-954.

Römheld V and Marschner H (1986) Evidence for a specific uptake system for iron phytosiderophores in roots of grasses. Plant Physiol 80:175-180.

Römheld V, Müller C and Marschner H (1984) Localization and capacity of proton pumps in roots of intact sunflower plants. Plant Physiol 76:603-606

Rost B and Valencia A (1996) Pitfalls of protein sequence analysis. Curr Opin Biotechnol 7:457-461.

Rost B (1999) Twilight zone of protein sequence alignments. Protein Eng 12:85-94.

Salamov AA and Solovyev VV (2000) Ab initio gene finding in Drosophila genomic DNA. Genome Res 10:516-522.

Shimamoto K and Kyozuka J (2001) Rice as a model for comparative genomics of plants. Annu Rev Plant Physiol Plant Mol Biol 53:399-419.

Silverstein KAT, Shoop E, Johnson JE, Kilian A, Freeman JL, Kunau TM, Awad IA, Mayer M and Retzel EF (2001) The MetaFam Server: a comprehensive protein family resource. Nucleic Acids Res 29:49-51.

Stephan UW and Scholtz G (1993) Nicotianamine: mediator of transport of iron and heavy metals in the phloem? Physiol Plant 88:522-529. 
Sugiura Y amd Nomoto K (1984) Phytosiderophores: structures and properties of mugineic acids and their metal complexes. Struct Bonding 58:107-135.

Swofford DL (2002) PAUP*. Phylogenetic Analysis Using Parsimony (*and Other Methods). Version 4. Sinauer Associates, Sunderland, MA.

Takahashi M, Nakanishi H, Kawasaki S, Nishizawa NK and Mori S (2001) Enhanced tolerance of rice to low iron availability in alkaline soils using barley nicotianamine aminotransferase genes. Nat Biotechnol 19:466-469.

Thomine S, Wang R, Ward JM, Crawford NM and Schroeder JI (2000) Cadmium and iron transport by members of a plant metal transporter family in Arabidopsis with homology to Nramp genes. Proc Natl Acad Sci USA 97:4991-4996.

Thompson JD, Higgins DG and Gibson TJ (1994) Clustal W: improving the sensitivity of progressive multiple sequence alignment through sequence weighting, position-specific gap penalties and weight matrix choice. Nucleic Acids Res 22:4673-4680.

Todd AE, Orengo CA and Thornton JM (2001) Evolution of function in protein superfamilies, from a structural perspective. $\mathrm{J}$ Mol Biol 307:1113-1143.

Tolay I, Erenoglu B, Römheld V, Braun HJ and Cakmak I (2001) Phytosiderophore release in Aegilops tauschii and Triticum species under zinc and iron deficiencies. J Exp Bot 52:1093-1099.

Torres MA, Onouchi H, Hamada S, Machida C, HammondKosack KE and Jones JD (1998) Six Arabidopsis thaliana homologues of the human respiratory burst oxidase (gp91phox). Plant J 14:365-370.

Vansuyt G, Mench M and Briat JF (2000) Soil-dependent variability of leaf iron accumulation in transgenic tobacco overexpressing ferritin. Plant Physiol Biochem 38:499-506.

Vert G, Briat JF and Curie C (2001) Arabidopsis IRT2 gene encodes a root-periphery iron transporter. Plant J 26:181-189.

Vert G, Grotz N, Dedaldechamp F, Gaymard F, Guerinot ML, Briat JF and Curie C (2002) IRT1, an Arabidopsis transporter essential for iron uptake from the soil and for plant growth. Plant Cell 14:1223-1233.

Walker EL, Burke T, Panaviene Z and Abbey PJ (2001) Possible roles for the Yellow Stripe-Like (YSL) family of proteins in the transport of metal-nicotianamine complexes. American Society of Plant Biologists Annual Meeting Abstracts. Minisymposium 1 - Membrane Transport. Abstract 11004 (also available at http://www.rycomusa.com/aspp2001/public/ M01/0986.html).

Waters BM, Blevins DG and Eide DJ (2002) Characterization of FRO1, a pea ferric-chelate reductase involved in root iron acquisition. Plant Physiol 129:85-94.

Waterston RH, Lander ES and Sulston JE (2002) On the sequencing of the human genome. Proc Natl Acad Sci USA 99:3712-3716.

Williams LE, Pittman JK and Hall JL (2000) Emerging mechanisms for heavy metal transport in plants. Biochim Biophys Acta 1465:104-126.

von Wirén N, Marschner H and Römheld V (1995) Uptake kinetics of iron-phytosiderophores in two maize genotypes differing in iron efficiency. Plant Physiol 93:611-616.

World Health Organization (2002) Nutrition. Reduction of iron deficiency anaemia. http://www.who.int/nut/ida.htm, 09/12/2002.

$\mathrm{Xu}$ Y and Uberbacher EC (1997) Automated gene identification in large-scale genomic sequences. J Comput Biol 4:325-338.

Yen M-R, Tseng YH and Saier Jr MH (2001) Maize Yellow Stripe1, an iron-phytosiderophore uptake transporter, is a member of the oligopeptide transporter (OPT) family. Microbiology 147:2881-2883.

Yu J, Hu S, Wang J, Wong G K-S, Li S, Liu B, Deng Y, Daí L, Zhou Y, Zhang X, Cao M, Liu J, Sun J, Tang J, Chen Y, Huang X, Lin W, Ye C, Tong W, Cong L, Geng J, Han Y, Li L, Li W, Hu G, Huang X, Li W, Jian Li, Liu Z, Li L, Liu J, Qi Q, Liu J, Li L, Li T, Wang X, Lu H, Wu T, Zhu M, Ni P, Han H, Dong W, Ren X, Feng X, Cui P, Li X, Wang H, Xu X, Zhai W, Xu Z, Zhang J, He S, Zhang J, Xu J, Zhang K, Zheng X, Dong J, Zeng W, Tao L, Ye J, Tan J, Ren X, Chen X, He J, Liu D, Tian W, Tian C, Xia H, Bao Q, Li G, Gao H, Cao T, Wang J, Zhao W, Li P, Chen W, Wang X, Zhang Y, Hu J, Wang J, Liu S, Yang J, Zhang G, Xiong Y, Li Z, Mao L, Zhou C, Zhu Z, Chen R, Hao B, Zheng W, Chen S, Guo W, Li G, Liu S, Tao M, Wang J, Zhu L, Yuan L and Yang H (2002) A draft sequence of the rice genome (Oryza sativa L. ssp. indica). Science 296:79-92.

Zhao H and Eide D (1996a) The yeast ZRT1 gene encodes the zinc transporter protein of a high-affinity uptake system induced by zinc limitation. Proc Natl Acad Sci USA 93:2454-2458.

Zhao H and Eide D (1996b) The ZRT2 gene encodes the low affinity zinc transporter in Saccharomyces cerevisiae. J Biol Chem 271:23203-23210.

Editor: Márcio de Castro Silva Filho 